\title{
One-loop quantum gravity in the Einstein universe
}

\author{
Ivan G. Avramidi and Samuel J. Collopy \\ Department of Mathematics, New Mexico Institute of Mining and Technology, \\ Socorro, NM 87801, U.S.A. \\ E-mail: iavramid@nmt.edu, samuel.collopy@gmail.com
}

ABStract: We study quantum gravity with the Einstein-Hilbert action including the cosmological constant on the Euclidean Einstein universe $S^{1} \times S^{3}$. We compute exactly the spectra and the heat kernels of the relevant operators on $S^{3}$ and use these results to compute the heat trace of the graviton and ghost operators and the exact one-loop effective action on $S^{1} \times S^{3}$. We show that the system is unstable in the infrared limit due to the presence of the negative modes of the graviton and the ghost operators. We study the thermal properties of the model with the temperature $T=\left(2 \pi a_{1}\right)^{-1}$ determined by the radius $a_{1}$ of the circle $S^{1}$. We show that the heat capacity $C_{v}$ is well defined and behaves like $\sim T^{3}$ in the high temperature limit and has a singularity of the type $\sim\left(T-T_{c}\right)^{-1}$, indicating a second-order phase transition, with the critical temperature $T_{c}$ determined by the cosmological constant $\Lambda$ and the radius $a$ of the sphere $S^{3}$. We also discuss some peculiar properties of the model such as the negative heat capacity as well as possible physical applications.

KEYwords: Models of Quantum Gravity, Nonperturbative Effects, Differential and Algebraic Geometry, Renormalization Group

ARXIV EPRINT: 1509.00929 


\section{Contents}

1 Introduction 1

2 One-loop Einstein gravity 2

$\begin{array}{lll}3 & \text { Heat trace coefficients } & 7\end{array}$

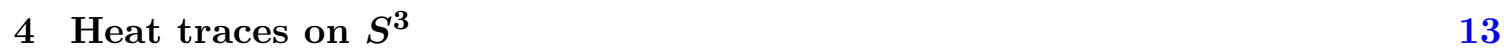

$\begin{array}{lll}4.1 & \text { Reduction to irreducible representations } & 13\end{array}$

$\begin{array}{lll}4.2 & \text { Heat trace for irreducible representations } & 14\end{array}$

$\begin{array}{lll}4.3 & \text { Heat trace of quantum gravity } & 16\end{array}$

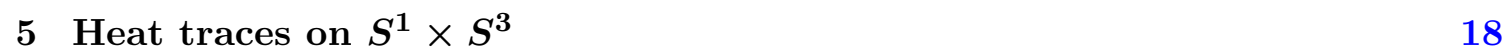

$\begin{array}{lll}5.1 & \text { Reduction of heat traces } & 18\end{array}$

$\begin{array}{lll}5.2 \text { Generators } & 20\end{array}$

5.3 Algebra of constant symmetric endomorphisms 21

5.4 Algebra of symmetric endomorphisms on $S^{3} \quad 24$

$\begin{array}{ll}5.5 \text { Group averages } & 27\end{array}$

5.6 Heat trace of operator $L_{1}$ on $S^{3} \quad 29$

5.7 Heat trace of the operator $L_{2}$ on $S^{3} \quad 30$

6 Effective action 31

$\begin{array}{lll}7 & \text { Thermodynamics } & 33\end{array}$

$\begin{array}{lll}8 & \text { Discussion } & 37\end{array}$

\section{Introduction}

The low-energy effective action in quantum field theory is a powerful tool that enables one to study the vacuum state of the theory. The low-energy effective action cannot be computed in the usual perturbation theory, and so to study it in the generic case, one needs new essentially non-perturbative methods. The development of such methods for the calculation of the heat kernel was initiated in our papers $[1,3]$ for a gauge theory in flat space, which were then applied to study the vacuum structure of the Yang-Mills theory in $[4,6]$. These ideas were first extended to scalar fields on curved manifolds in $[2,5]$ and finally to arbitrary twisted spin-tensor fields in [8]. In [9] we applied these methods to study quantum gravity and Yang-Mills theory on any symmetric space. Further, we applied these methods to study the thermal Yang-Mills theory on product of spheres, such as $S^{1} \times S^{1} \times S^{2}$ and $S^{1} \times S^{3}$ in $[11,12]$. 
In the present paper we apply these methods to study the one-loop low-energy effective action in quantum Einstein general relativity in the Einstein Universe background at finite temperature. From the mathematical point of view, we compute the one-loop effective action for the Einsten-Hilbert action with cosmological constant on the background $S^{1} \times S^{3}$.

This paper is organized as follows. In section 2 we introduce all the relevant operators for the calculation of the one-loop effective action in Einstein quantum gravity. We refer to the paper [9] for the details. In section 3 we compute the heat trace coefficients. In Sec 4 . we study the quantum gravity on $S^{3}$ and compute all relevant heat traces on $S^{3}$. We refer to the paper [12] for the details of the calculation of the heat traces on $S^{3}$ for any representation. In section 5 we compute the heat traces and the effective action on $S^{1} \times S^{3}$. Finally, in section 6 we discuss the thermodynamic properties of the model.

\section{One-loop Einstein gravity}

In this section we follow our previous work $[9,10]$. The dynamics of the gravitational field parametrized by the Riemannian metric on a closed (compact without boundary) manifold $(M, g)$ of dimension $n$ is described by the Hilbert-Einstein action of general relativity, which (in Euclidean formulation) has the form

$$
S=\frac{1}{16 \pi G} \int_{M} d x g^{1 / 2}(-R+2 \Lambda),
$$

where $g=\operatorname{det} g_{\mu \nu}, G$ is the gravitational constant and $\Lambda$ is the cosmological constant. The classical vacuum Einstein equations are determined by the first variation of the action

$$
16 \pi G g^{-1 / 2} \frac{\delta S}{\delta g_{\mu \nu}}=R^{\mu \nu}-\frac{1}{2} R g^{\mu \nu}+\Lambda g^{\mu \nu}=0 .
$$

In two dimensions the action is trivial

$$
S=\frac{1}{16 \pi G}\{-4 \pi \chi(M)+2 \Lambda \operatorname{vol}(M)\},
$$

where $\chi(M)$ is the Euler characteristic of the manifold $M$ and $\operatorname{vol}(M)$ is its volume. Therefore, it does not have any extremal metrics; more precisely, in two dimensions every metric satisfies the Einstein equations with zero cosmological constant,

$$
R_{a b}=\frac{1}{2} R g_{a b},
$$

and, therefore, the Einstein equations do not have any solutions for any $\Lambda \neq 0$, which means that Einstein gravity in two dimensions is purely topological.

For this reason, we restrict ourselves to $n>2$. In this case the Riemann tensor can be decomposed as follows

$$
R_{c d}^{a b}=C^{a b}{ }_{c d}+\frac{4}{n-2} R_{\left[{ }^{2} \delta^{b]}\right]}^{[a}-\frac{2}{(n-1)(n-2)} R \delta^{[a}{ }_{\left[{ } \delta^{b]} d\right]},
$$


where $C_{a b c d}$ is the Weyl tensor. The norm of the Riemann tensor is then

$$
R_{a b c d} R^{a b c d}=C_{a b c d} C^{a b c d}+\frac{4}{n-2} R_{a b} R^{a b}-\frac{2}{(n-1)(n-2)} R^{2} .
$$

The solutions of the Einstein equations determine the Einstein spaces,

$$
R_{a b}=\frac{2}{n-2} \Lambda g_{a b}
$$

and, therefore,

$$
R=\frac{2 n}{n-2} \Lambda
$$

In this case the Riemann tensor is

$$
R_{c d}^{a b}=C_{c d}^{a b}+\frac{4}{(n-1)(n-2)} \Lambda \delta_{\left[{ }^{[a} \delta_{d]}^{b]}\right.},
$$

with the norm

$$
R_{a b c d} R^{a b c d}=C_{a b c d} C^{a b c d}+\frac{8 n}{(n-1)(n-2)^{2}} \Lambda^{2} .
$$

The case of three dimensions is special. In this case the Weyl tensor is equal to zero identically, and, therefore, the Riemann tensor is fully determined by the Ricci tensor,

$$
\left.R^{a b}{ }_{c d}=4 R_{\left[c^{[a} \delta^{b]}\right]}-R \delta^{[a}{ }_{[c} \delta^{b]} d\right] .
$$

Therefore, in particular,

$$
R_{a b c d} R^{a b c d}-4 R_{a b} R^{a b}+R^{2}=0 .
$$

The Einstein equations take the form

$$
R_{a b}=2 \Lambda g_{a b}
$$

and, therefore, the curvature tensor of Einstein spaces is fully determined by the metric,

$$
\left.R_{c d}^{a b}=2 \Lambda \delta^{[a}{ }_{[{ }} \delta^{b]} d\right] .
$$

This means that the only Einstein spaces in three dimensions are the (locally) maximally symmetric spaces, the sphere $S^{3}$ for $\Lambda>0$, the hyperbolic manifolds $H^{3} / \Gamma$ for $\Lambda<0$, where $\Gamma$ is a lattice in $S O^{+}(1,3)$; for $\Lambda=0$ the only solutions are flat manifolds, like a torus $T^{3}$. In any case, gravity in three dimensions is rigid, that is, it does not have any propagating degrees of freedom.

Notice that the same invariant (2.12) plays a role in higher dimensions as well. In particular, in dimension $n=4$ the integral of that invariant determines the Euler characteristic of the manifold

$$
\begin{aligned}
\chi(M) & =\frac{1}{32 \pi^{2}} \int_{M} d x g^{1 / 2}\left(R_{a b c d} R^{a b c d}-4 R_{a b} R^{a b}+R^{2}\right) \\
& =\frac{1}{32 \pi^{2}} \int_{M} d x g^{1 / 2}\left(C_{a b c d} C^{a b c d}-2 R_{a b} R^{a b}+\frac{2}{3} R^{2}\right) .
\end{aligned}
$$


When the Einstein equations (2.7), (2.8), are satisfied the Ricci tensor is determined by the metric. That is, for $n=4$,

$$
R_{a b}=\Lambda g_{a b}, \quad R=4 \Lambda,
$$

and the integral norm of the Riemann tensor is determined by the Euler characteristic

$$
\begin{aligned}
\chi(M) & =\frac{1}{32 \pi^{2}} \int_{M} d x g^{1 / 2} R_{a b c d} R^{a b c d} \\
& =\frac{1}{32 \pi^{2}} \int_{M} d x g^{1 / 2}\left(C_{a b c d} C^{a b c d}+\frac{8}{3} \Lambda^{2}\right) .
\end{aligned}
$$

Note that the Euler characteristic of Einstein spaces in four dimensions is positive definite. It is worth stressing that this disagrees with eq. (115) in [18].

The diffeomorphism invariance of the Einstein-Hilbert functional means that the metric carries some non-physical (gauge) degrees of freedom described by a vector field. In $n$ dimensions a vector field has $n$ independent components and a symmetric 2 -tensor field has $n(n+1) / 2$ independent components. Therefore, the gravitational field in $n$ dimensions has

$$
N(n)=\frac{n(n+1)}{2}-2 n=\frac{n(n-3)}{2}
$$

degrees of freedom. This number is equal to $N(4)=2$ in four dimensions as expected; however, it vanishes in three dimensions, $N(3)=0$. In two dimensions it gives a meaningless result, $N(2)=-1$. We will compute the effective action in three dimensions below but one should realize that in three dimensions the Einstein gravity does not have any dynamics $[19,20]$.

One of the fundamental problems of quantum Einstein gravity is that the Euclidean Einstein-Hilbert action is unbounded from below, which leads to the divergence of the Euclidean path integral over all metrics. This divergence is conceptual in nature and is much more serious than the usual ultraviolet divergence of the quantum field theory. It is well known [18] that under a conformal transformation

$$
\bar{g}_{\mu \nu}=\omega^{4 /(n-2)} g_{\mu \nu},
$$

where $\omega$ is a smooth positive function on $M$, the action takes the form

$$
S=\frac{1}{16 \pi G} \frac{8(n-1)}{(n-2)} \int_{M} d x g^{1 / 2}\left\{-\frac{1}{2} \omega Y \omega+\frac{(n-2)}{4(n-1)} \Lambda \omega^{2 n /(n-2)}\right\},
$$

where $Y$ is the Yamabe operator

$$
Y=-\Delta+\frac{n-2}{4(n-1)} R .
$$

The Yamabe operator is nothing but the conformally covariant scalar Laplacian. It is a selfadjoint elliptic partial differential operator with a positive leading symbol. The spectrum of 
such operator is real, discrete, and with finite multiplicities; it is bounded from below and unbounded from above. This shows that the action functional is unbounded from below. It is obvious that by keeping the metric $g_{\mu \nu}$ constant and taking the function $\omega$ to be bounded and increasingly oscillating the action can be made arbitrarily large and negative. This is a well known conformal problem of quantum gravity. It has been suggested [18] that this problem can be avoided by deforming the contour of integration in the path integral over the conformal factor to make it purely imaginary, which will turn the action into a standard functional of quantum field theory. However, such an approach cannot be taken seriously. This is a major problem of Einstein quantum gravity and it remains open. A solution to this problem would require a modification of the Einstein-Hilbert action but we do not attempt to solve it in the present paper.

The standard loop expansion of the Euclidean effective action has the form

$$
\Gamma=S+\hbar \Gamma_{(1)}+O\left(\hbar^{2}\right)
$$

where $\Gamma_{(1)}$ is the one-loop effective action. The one-loop effective action is determined by the graviton operator $L_{2}$ acting on symmetric two-tensor fields and the Faddeev-Popov ghost operator $L_{1}$ acting on vector fields. In the Euclidean formulation the zeta-regularized one-loop effective action has the form

$$
\Gamma_{(1)}=-\frac{1}{2} \zeta_{G R}^{\prime}(0)
$$

where

$$
\zeta_{\mathrm{GR}}(s)=\zeta_{L_{2}}(s)-2 \zeta_{L_{1}}(s),
$$

and $\zeta_{L_{1}}(s)$ and $\zeta_{L_{2}}(s)$ are the zeta functions of the operators $L_{1}$ and $L_{2}$ defined by

$$
\zeta_{L}(s)=\frac{\mu^{2 s}}{\Gamma(s)} \int_{0}^{\infty} d t t^{s-1} e^{-t z^{2}} \Theta_{L}(t)
$$

where

$$
\Theta_{L}(t)=\operatorname{Tr} \exp (-t L) .
$$

The renormalization parameter $\mu$ is introduced to preserve dimensions and $z$ is a sufficiently large infra-red regularization parameter, which should be set to zero at the end of the calculation. Therefore,

$$
\zeta_{\mathrm{GR}}(s)=\frac{\mu^{2 s}}{\Gamma(s)} \int_{0}^{\infty} d t t^{s-1} e^{-t z^{2}} \Theta_{\mathrm{GR}}(t)
$$

where

$$
\Theta_{\mathrm{GR}}(t)=\Theta_{L_{2}}(t)-2 \Theta_{L_{1}}(t)
$$

we will call this invariant the heat trace of quantum gravity.

The operators $L_{2}$ and $L_{1}$ are determined by the second variation of the action and then by imposing some gauge condition on the metric fluctuation (see, for example, $[7,15]$ ). The 
second variation of the action defines a second-order partial differential operator $P$ acting on symmetric two-tensors by

$$
16 \pi G g^{-1 / 2} \frac{\delta^{2} S}{\delta g_{\mu \nu} \delta g_{\alpha \beta}} h_{\alpha \beta}=\frac{1}{2} P^{\mu \nu \alpha \beta} h_{\alpha \beta},
$$

where

$$
\begin{aligned}
P^{\mu \nu, \alpha \beta}= & -\left(g^{\alpha(\mu} g^{\nu) \beta}-g^{\alpha \beta} g^{\mu \nu}\right) \Delta \\
& -g^{\mu \nu} \nabla^{(\alpha} \nabla^{\beta)}-g^{\alpha \beta} \nabla^{(\mu} \nabla^{\nu)}+2 \nabla^{(\mu} g^{\nu)(\alpha} \nabla^{\beta)} \\
& -2 R^{(\mu|\alpha| \nu) \beta}-g^{\alpha(\mu} R^{\nu) \beta}-g^{\beta(\mu} R^{\nu) \alpha}+R^{\mu \nu} g^{\alpha \beta}+R^{\alpha \beta} g^{\mu \nu} \\
& +\left(g^{\mu(\alpha} g^{\beta) \nu}-\frac{1}{2} g^{\mu \nu} g^{\alpha \beta}\right)(R-2 \Lambda) .
\end{aligned}
$$

In the minimal gauge the non-diagonal derivatives in both the graviton operator and the ghost operator vanish and the operators take the form

$$
\begin{aligned}
\tilde{L}_{2}{ }^{c d, a b}= & \left(g^{a(c} g^{d) b}-\frac{1}{2} g^{a b} g^{c d}\right)(-\Delta+R-2 \Lambda) \\
& -2 R^{(c|a| d) b}-g^{a(c} R^{d) b}-g^{b(c} R^{d) a)}+R^{c d} g^{a b}+g^{c d} R^{a b}, \\
\tilde{L}_{1}^{a b}= & -g^{a b} \Delta-R^{a b} .
\end{aligned}
$$

We should stress that the operator $\tilde{L}_{2}$ differs from the eq. (16.37) in [14].

The tensor

$$
E^{c d, a b}=g^{a(c} g^{d) b}-\frac{1}{2} g^{a b} g^{c d}
$$

here is the metric in the space of symmetric tensors. It is easy to see that it is positive definite in the subspace of traceless symmetric tensors but it is negative definite in the conformal (scalar) sector. This is exactly the problem of the conformal mode in quantum gravity discussed above. Following the standard approach $[13-15,18]$ we simply assume that it can be fixed somehow by some physical arguments and proceed as follows. We factor out this metric from the operator $\tilde{L}_{2}$ to define the graviton operator $L_{2}$ and the ghost operator $L_{1}$ in the canonical Laplace-type form

$$
L_{j}=-\Delta+Q_{j},
$$

where the potentials for both operators are [9]

$$
\begin{aligned}
\left(Q_{1}\right)_{b}^{a}= & -R_{b}^{a}, \\
\left(Q_{2}\right)^{c d}{ }_{a b}= & -2 R_{\left({ }_{a}{ }^{d} b\right)}-2 \delta_{(a}^{(c} R_{b)}^{d)}+R^{c d} g_{a b}+\frac{2}{n-2} g^{c d} R_{a b} \\
& -\frac{1}{(n-2)} g^{c d} g_{a b} R+\delta^{c}{ }_{(a} \delta_{b)}^{d}(R-2 \Lambda) .
\end{aligned}
$$

We should stress here that the endomorphism $Q_{2}$ does not coincide with the eq. (16.78) in $[14]$. 
It is well known that the heat trace of Laplace type operators has the asymptotic expansion as $t \rightarrow 0$

$$
\Theta_{L}(t) \sim(4 \pi t)^{-n / 2} \sum_{k=0}^{\infty} t^{k} B_{k}(L),
$$

where $B_{k}(L)$ are the so-called Hadamard-Minakshisundaram-DeWitt-Seeley coefficients (or simply heat trace coefficients) of the operator $L$. This means that the function $\Theta(t)$ has similar asymptotic expansion as $t \rightarrow 0$

$$
\Theta_{\mathrm{GR}}(t) \sim(4 \pi t)^{-n / 2} \sum_{k=0}^{\infty} t^{k} C_{k}
$$

where

$$
C_{k}=B_{k}\left(L_{2}\right)-2 B_{k}\left(L_{1}\right) .
$$

It is easy to find the dependence of the effective action on the renormalization parameter; by integrating the equation

$$
\mu \frac{\partial}{\partial \mu} \Gamma_{(1)}=-\zeta_{\mathrm{GR}}(0)
$$

we get

$$
\Gamma_{(1)}(\mu)=\Gamma_{(1)}\left(\mu_{0}\right)-\log \left(\frac{\mu}{\mu_{0}}\right) \zeta_{\mathrm{GR}}(0) .
$$

This enables one to study the high-energy asymptotics of the effective action as $\mu \rightarrow \infty$.

For the Laplace type operators the value of the zeta function at $s=0$ is determined by a specific heat trace coefficient

$$
\zeta_{L}(0)= \begin{cases}(4 \pi)^{-n / 2} B_{n / 2}(L), & \text { for even } n \\ 0, & \text { for odd } n\end{cases}
$$

Therefore,

$$
\zeta_{\mathrm{GR}}(0)= \begin{cases}(4 \pi)^{-n / 2} C_{n / 2}, & \text { for even } n \\ 0, & \text { for odd } n\end{cases}
$$

in particular, in four dimensions, $n=4$,

$$
\zeta_{\mathrm{GR}}(0)=(4 \pi)^{-2} C_{2}
$$

\section{Heat trace coefficients}

We will need the heat trace coefficients $B_{0}, B_{1}$ and $B_{2}$ for the operators $L_{1}$ and $L_{2}$. They have the following well-known form $[7,17]$ (we neglected the inessential total derivatives 
here which do not contribute to the global invariants)

$$
\begin{aligned}
& B_{0}(L)= \int_{M} d x g^{1 / 2} \operatorname{tr} I, \\
& B_{1}(L)=\int_{M} d x g^{1 / 2} \operatorname{tr}\left(\frac{1}{6} R I-Q\right), \\
& B_{2}(L)=\int_{M} d x g^{1 / 2} \operatorname{tr}\left\{\frac{1}{2} Q^{2}-\frac{1}{6} R Q+\frac{1}{12} \mathcal{R}_{a b} \mathcal{R}^{a b}\right. \\
& \\
&\left.\qquad I\left(\frac{1}{72} R^{2}+\frac{1}{180} R_{a b c d} R^{a b c d}-\frac{1}{180} R_{a b} R^{a b}\right)\right\} .
\end{aligned}
$$

Here $I$ is the identity endomorphism and $\mathcal{R}_{a b}$ is the curvature of the spin connection of a tensor field realizing a representation of the spin group defined by

$$
\mathcal{R}_{\mu \nu}=\frac{1}{2} R^{a b}{ }_{\mu \nu} \Sigma_{a b},
$$

where $\Sigma_{a b}$ are the generators of the spin group $\operatorname{Spin}(n)$ satisfying the commutation relations

$$
\left[\Sigma_{a b}, \Sigma_{c d}\right]=-g_{a c} \Sigma_{b d}+g_{b c} \Sigma_{a d}+g_{a d} \Sigma_{b c}-g_{b d} \Sigma_{a c} .
$$

For the vector representation the identity and the generators have the form

$$
\begin{aligned}
\left(I_{1}\right)^{c}{ }_{d} & =\delta^{c}{ }_{d}, \\
\left(\Sigma_{(1), a b}\right)^{c}{ }_{d} & =2 \delta^{c}{ }_{[a} g_{b] d},
\end{aligned}
$$

Therefore, $\operatorname{tr} I_{1}=n$ and

$$
B_{0}\left(L_{1}\right)=n \operatorname{vol}(M)
$$

Also, we have

$$
\operatorname{tr} Q_{1}=-R,
$$

and, therefore,

$$
B_{1}\left(L_{1}\right)=\int_{M} d x g^{1 / 2} \frac{1}{6}(n+6) R .
$$

Further, we compute

$$
\left.\operatorname{tr} \Sigma_{(1), a b} \Sigma_{(1)}^{p q}=-4 \delta_{[a}^{[p} \delta^{q]} b\right]
$$

and

$$
\operatorname{tr} \mathcal{R}_{(1), a b} \mathcal{R}_{(1)}^{a b}=-R_{a b c d} R^{a b c d} .
$$


We also have

$$
\operatorname{tr}\left(Q_{1}\right)^{2}=R_{a b} R^{a b}
$$

and, therefore,

$$
B_{2}\left(L_{1}\right)=\int_{M} d x g^{1 / 2}\left\{\frac{n-15}{180} R_{a b c d} R^{a b c d}+\frac{90-n}{180} R_{a b} R^{a b}+\frac{n+12}{72} R^{2}\right\} .
$$

Next, we compute the heat trace coefficients for the operator $L_{2}$. For the tensor representation the identity and the generators have the form

$$
\begin{aligned}
\left(I_{2}\right)^{e f}{ }_{c d} & \left.=\delta^{(e}{ }_{(c} \delta^{f)} d\right), \\
\left(\Sigma_{(2), a b}\right)^{e f}{ }_{c d} & \left.=4 \delta_{[a}^{(e} g_{b]}{ }_{(c} \delta^{f)} d\right) .
\end{aligned}
$$

First, we have $\operatorname{tr} I_{2}=n(n+1) / 2$, hence,

$$
B_{0}\left(L_{2}\right)=\frac{1}{2} n(n+1) \operatorname{vol}(M) .
$$

Now, we introduce the following endomorphisms

$$
\begin{aligned}
& \left(V_{1}\right)^{c d}{ }_{a b}=R_{(a b)}^{c}, \\
& \left.\left(V_{2}\right)^{c d}{ }_{a b}=\delta_{(a}^{(c} R^{d)} b\right), \\
& \left(V_{3}\right)^{c d}{ }_{a b}=R^{c d} g_{a b}, \\
& \left(V_{4}\right)^{c d}{ }_{a b}=g^{c d} R_{a b}, \\
& \left(V_{5}\right)^{c d}{ }_{a b}=g^{c d} g_{a b} .
\end{aligned}
$$

Then the endomorphism $Q_{2}$ has the form

$$
Q_{2}=\tilde{Q}_{2}+(R-2 \Lambda) I_{2},
$$

where

$$
\tilde{Q}_{2}=-2 V_{1}-2 V_{2}+V_{3}+\frac{2}{n-2} V_{4}-\frac{1}{(n-2)} R V_{5} .
$$

Now, by using the traces

$$
\begin{aligned}
& \operatorname{tr} V_{1}=-\frac{1}{2} R, \\
& \operatorname{tr} V_{2}=\frac{1}{2}(n+1) R, \\
& \operatorname{tr} V_{3}=R, \\
& \operatorname{tr} V_{4}=R, \\
& \operatorname{tr} V_{5}=n .
\end{aligned}
$$

we compute

$$
\operatorname{tr} \tilde{Q}_{2}=-n R,
$$


and, therefore,

$$
\operatorname{tr} Q_{2}=\frac{1}{2} n(n-1) R-n(n+1) \Lambda
$$

which gives

$$
B_{1}\left(L_{2}\right)=\int_{M} d x g^{1 / 2}\left\{-\frac{1}{12} n(5 n-7) R+n(n+1) \Lambda\right\}
$$

Now, we have

$$
\operatorname{tr}\left(Q_{2}\right)^{2}=\operatorname{tr}\left(\tilde{Q}_{2}\right)^{2}+\frac{1}{2} n(n-3) R^{2}-2 n(n-1) R \Lambda+2 n(n+1) \Lambda^{2} .
$$

Next, by using the traces of the products

$$
\begin{aligned}
& \operatorname{tr} V_{1} V_{1}=\frac{3}{4} R_{a b c d} R^{a b c d} \\
& \operatorname{tr} V_{2} V_{2}=\frac{1}{4}(n+2) R_{a b} R^{a b}+\frac{1}{4} R^{2} \\
& \operatorname{tr} V_{3} V_{3}=R^{2} \\
& \operatorname{tr} V_{4} V_{4}=R^{2} \\
& \operatorname{tr} V_{5} V_{5}=n^{2} \\
& \operatorname{tr} V_{1} V_{2}=-\frac{1}{2} R_{a b} R^{a b} \\
& \operatorname{tr} V_{1} V_{3}=R_{a b} R^{a b} \\
& \operatorname{tr} V_{1} V_{4}=R_{a b} R^{a b} \\
& \operatorname{tr} V_{1} V_{5}=R \\
& \operatorname{tr} V_{2} V_{3}=R_{a b} R^{a b} \\
& \operatorname{tr} V_{2} V_{4}=R_{a b} R^{a b} \\
& \operatorname{tr} V_{2} V_{5}=R \\
& \operatorname{tr} V_{3} V_{4}=n R_{a b} R^{a b} \\
& \operatorname{tr} V_{3} V_{5}=n R \\
& \operatorname{tr} V_{4} V_{5}=n R
\end{aligned}
$$

we obtain

$$
\operatorname{tr}\left(\tilde{Q}_{2}\right)^{2}=3 R_{a b c d} R^{a b c d}+\frac{n^{2}-8 n+4}{n-2} R_{a b} R^{a b}+\frac{n+2}{n-2} R^{2} .
$$

By using these results we get

$$
\begin{aligned}
\operatorname{tr}\left(Q_{2}\right)^{2}= & 3 R_{a b c d} R^{a b c d}+\frac{n^{2}-8 n+4}{n-2} R_{a b} R^{a b}+\frac{n^{3}-5 n^{2}+8 n+4}{2(n-2)} R^{2} \\
& -2 n(n-1) R \Lambda+2 n(n+1) \Lambda^{2} .
\end{aligned}
$$


We introduce yet another endomorphism

$$
\left(T_{p q}\right)^{c d}{ }_{a b}=\delta_{[p}^{(c} g_{q](a} \delta^{d)}{ }_{b)} .
$$

By using

$$
\left.\operatorname{tr} T_{p q} T^{r s}=-\frac{1}{4}(n+2) \delta^{[r}{ }_{[p} \delta^{s]}\right]
$$

we get

$$
\operatorname{tr} \Sigma_{(2), a b} \Sigma_{(2)}^{p q}=-4(n+2) \delta_{[a}^{[p} \delta_{b]}^{q]}
$$

and, therefore,

$$
\operatorname{tr} \mathcal{R}_{(2), a b} \mathcal{R}_{(2)}{ }^{a b}=-(n+2) R_{a b c d} R^{a b c d} .
$$

By using these results we get

$$
B_{2}\left(L_{2}\right)=\int_{M} d x g^{1 / 2}\left\{\alpha_{1} R_{a b c d} R^{a b c d}+\alpha_{2} R_{a b} R^{a b}+\alpha_{3} R^{2}+\gamma_{1} R \Lambda+\gamma_{2} \Lambda^{2}\right\},
$$

where

$$
\begin{aligned}
& \alpha_{1}=\frac{1}{360}\left(n^{2}-29 n+480\right), \\
& \alpha_{2}=\frac{-n^{3}+181 n^{2}-1438 n+720}{360(n-2)} \\
& \alpha_{3}=\frac{25 n^{3}-145 n^{2}+262 n+144}{144(n-2)} \\
& \gamma_{1}=\frac{1}{6} n(7-5 n) \\
& \gamma_{2}=n(n+1)
\end{aligned}
$$

Finally, by using these results we compute the coefficients $C_{k},(2.39)$,

$$
\begin{aligned}
& C_{0}=\frac{1}{2} n(n-3) \operatorname{vol}(M), \\
& C_{1}=\int_{M} d x g^{1 / 2}\left\{-\frac{1}{12}\left(5 n^{2}-3 n+24\right) R+n(n+1) \Lambda\right\}, \\
& C_{2}=\int_{M} d x g^{1 / 2}\left\{\beta_{1} R_{a b c d} R^{a b c d}+\beta_{2} R_{a b} R^{a b}+\beta_{3} R^{2}+\gamma_{1} R \Lambda+\gamma_{2} \Lambda^{2}\right\},
\end{aligned}
$$

where

$$
\begin{aligned}
& \beta_{1}=\frac{1}{360}\left(n^{2}-33 n+540\right), \\
& \beta_{2}=\frac{-n^{3}+185 n^{2}-1806 n+1440}{360(n-2)}, \\
& \beta_{3}=\frac{25 n^{3}-149 n^{2}+222 n+240}{144(n-2)} .
\end{aligned}
$$

These results are also different from the ones given by eqs. (16.79)-(16.81) of [14]. 
We will need these coefficients for $n=4$. By using the above results we obtain in this case

$$
\begin{aligned}
& B_{0}\left(L_{1}\right)=4 \operatorname{vol}(M), \\
& B_{1}\left(L_{1}\right)=\int_{M} d x g^{1 / 2} \frac{5}{3} R \\
& B_{2}\left(L_{1}\right)=\int_{M} d x g^{1 / 2}\left\{-\frac{11}{180} R_{a b c d} R^{a b c d}+\frac{43}{90} R_{a b} R^{a b}+\frac{2}{9} R^{2}\right\}, \\
& B_{0}\left(L_{2}\right)=10 \operatorname{vol}(M), \\
& B_{1}\left(L_{2}\right)=\int_{M} d x g^{1 / 2}\left\{-\frac{13}{3} R+20 \Lambda\right\}, \\
& B_{2}\left(L_{2}\right)=\int_{M} d x g^{1 / 2}\left\{\frac{19}{18} R_{a b c d} R^{a b c d}-\frac{55}{18} R_{a b} R^{a b}+\frac{59}{36} R^{2}-\frac{26}{3} R \Lambda+20 \Lambda^{2}\right\} .
\end{aligned}
$$

Therefore, we obtain the total coefficients (2.39)

$$
\begin{aligned}
C_{0} & =2 \operatorname{vol}(M), \\
C_{1} & =\int_{M} d x g^{1 / 2}\left\{-\frac{23}{3} R+20 \Lambda\right\}, \\
C_{2} & =\int_{M} d x g^{1 / 2}\left\{\frac{53}{45} R_{a b c d} R^{a b c d}-\frac{361}{90} R_{a b} R^{a b}+\frac{43}{36} R^{2}-\frac{26}{3} R \Lambda+20 \Lambda^{2}\right\} \\
& =\frac{1696}{45} \pi^{2} \chi(M)+\int_{M} d x g^{1 / 2}\left\{-\frac{1}{90} R_{a b} R^{a b}+\frac{7}{36} R^{2}-\frac{26}{3} R \Lambda+20 \Lambda^{2}\right\} .
\end{aligned}
$$

Finally, when the Einstein equations are satisfied these coefficients in four dimensions take the form

$$
\begin{aligned}
C_{0} & =2 \operatorname{vol}(M), \\
C_{1} & =-\frac{32}{3} \Lambda \operatorname{vol}(M), \\
C_{2} & =\frac{1696}{45} \pi^{2} \chi(M)-\frac{58}{5} \Lambda^{2} \operatorname{vol}(M) .
\end{aligned}
$$

This gives the quantity

$$
\zeta_{\mathrm{GR}}(0)=\frac{106}{45} \chi(M)-\frac{29}{40} \frac{\Lambda^{2}}{\pi^{2}} \operatorname{vol}(M)
$$

determining the scaling properties of the model. It is worth stressing that this result coincides with the eq. (4.23) of [13] and disagrees with the results of [18], eq. (79), and [16], where the second coefficient is $657 / 540$ instead of $-29 / 40$; in particular, it is not positive definite contrary to the claim of [18]. 


\section{Heat traces on $S^{3}$}

\subsection{Reduction to irreducible representations}

In this section we compute the effective action and the relevant heat traces on the 3 -sphere $S^{3}$ of radius $a$. We define the dimensionless cosmological constant by

$$
\lambda=a^{2} \Lambda
$$

The curvature in the orthonormal frame has the form

$$
\begin{aligned}
R_{c d}^{a b} & =\frac{1}{a^{2}} \varepsilon^{f a b} \varepsilon_{f c d}=\frac{1}{a^{2}}\left(\delta_{c}^{a} \delta_{d}^{b}-\delta_{d}^{a} \delta_{c}^{b}\right), \\
R_{a b} & =\frac{2}{a^{2}} \delta_{a b}, \\
R & =\frac{6}{a^{2}} .
\end{aligned}
$$

Here and below $\varepsilon_{a b c}$ is the three-dimensional Levi-Civita symbol.

The volume of the sphere is

$$
\operatorname{vol}\left(S^{3}\right)=2 \pi^{2} a^{3}
$$

and the Euclidean classical Einstein-Hilbert action on $S^{3}$ is equal to

$$
S=\frac{\pi}{4 G} a\left(-3+a^{2} \Lambda\right) .
$$

Note that the classical action is bounded from below and attains a minimum equal to

$$
S_{0}=-\frac{\pi}{2 G \sqrt{\Lambda}}
$$

at the radius determined by the cosmological constant

$$
a_{0}=\Lambda^{-1 / 2}
$$

so that classically the dimensionless cosmological constant is equal to $1, \lambda_{0}=1$. We will compute the heat trace of the Laplacian for the unit sphere $S^{3}$ by setting $a=1$; the trivial dimensional factor $a$ can be easily restored at the end of the calculation by replacing $t \mapsto t / a^{2}$.

Let

$$
\left(\Pi_{0}\right)^{a b}{ }_{c d}=\frac{1}{3} g^{a b} g_{c d}
$$

be the projection to the scalar representation and

$$
\Pi_{2}=I_{2}-\Pi_{0}
$$

be the projection onto the space of traceless symmetric tensors. Note that the projection $\Pi_{2}$ acts as identity in the subspace of traceless symmetric tensors. In three dimensions, the dimensions of these subspaces are

$$
\begin{aligned}
\operatorname{tr} I_{1} & =3, & \operatorname{tr} I_{2} & =6, \\
\operatorname{tr} \Pi_{0} & =1, & \operatorname{tr} \Pi_{2} & =5 .
\end{aligned}
$$


This is consistent with the dimension of the general irreducible representation labeled by an integer $j$,

$$
\operatorname{tr}_{j} \Pi_{j}=2 j+1
$$

Then the potential terms are

$$
\begin{aligned}
& Q_{1}=-2 I_{1}, \\
& Q_{2}=(4-2 \lambda) \Pi_{2}-(2+2 \lambda) \Pi_{0} .
\end{aligned}
$$

We will reduce the calculation of the heat traces of the operators $L_{1}$ and $L_{2}$ to the calculation of the heat trace on the unit sphere $S^{3}$ of pure Laplacians $\Delta_{j}$ acting on irreducible representations $j$,

$$
\Theta_{j}(t)=\operatorname{Tr} \exp \left(t \Delta_{j}\right) .
$$

First of all, we immediately see that since the endomorphism $Q_{1}$ is constant, we have

$$
\exp \left(-t Q_{1}\right)=e^{2 t} I_{1}
$$

and, therefore, the heat trace of the ghost operator is

$$
\Theta_{L_{1}}(t)=e^{2 t} \Theta_{1}(t)
$$

We also have a similar formula for the operator $L_{2}$,

$$
\Theta_{L_{2}}(t)=\operatorname{Tr} \exp \left(-t Q_{2}\right) \exp (t \Delta)
$$

However, the general tensor representation contains the irreducible representation with $j=2$ (traceless symmetric two-tensors) and the scalar representation with $j=0$ (trace). The space of symmetric tensors decomposes canonically into the direct sum of the traceless tensors and scalars with the corresponding projections $\Pi_{2}$ and $\Pi_{0}$. It is easy to see that

$$
\exp \left(-t Q_{2}\right)=e^{(-4+2 \lambda) t} \Pi_{2}+e^{(2+2 \lambda) t} \Pi_{0}
$$

Therefore, the heat trace of the graviton operator takes the form

$$
\Theta_{L_{2}}(t)=e^{(-4+2 \lambda) t} \Theta_{2}(t)+e^{(2+2 \lambda) t} \Theta_{0}(t) .
$$

\subsection{Heat trace for irreducible representations}

Because the graviton operator neatly splits, we only need to compute the heat traces for Laplacians in irreducible representation $j$ for integer $j$. This heat trace can be computed by using the heat kernel diagonal for the Laplacian $\Delta_{j}$ on the unit sphere $S^{3}$ given by the eq. (6.16) of our paper [12]. To get the heat trace we have to multiply the heat kernel diagonal by the volume of the sphere $S^{3}$ equal to $\operatorname{vol}\left(S^{3}\right)=2 \pi^{2}$ and by the dimension of the representation $j$ equal to $(2 j+1)$. This gives

$$
\Theta_{j}(t)=\frac{\sqrt{\pi}}{4} t^{-3 / 2} e^{t[j(j+1)+1]} \sum_{n=-\infty}^{\infty} \sum_{|\mu| \leq j} \exp \left(-\frac{\pi^{2} n^{2}}{t}-\mu^{2} t\right)\left(1-2 \mu^{2} t-\frac{2 \pi^{2} n^{2}}{t}\right)
$$


Following [12] we introduce the function

$$
\Omega(t)=\sum_{n=-\infty}^{\infty} \exp \left(-\frac{n^{2} \pi^{2}}{t}\right)
$$

which can be expressed in terms of the Jacobi theta function

$$
\Omega(t)=\theta_{3}\left(0, e^{-\pi^{2} / t}\right),
$$

and satisfies the following duality relation

$$
\Omega(t)=\sqrt{\frac{t}{\pi}} \Omega\left(\frac{\pi^{2}}{t}\right)=\sqrt{\frac{t}{\pi}} \sum_{n=-\infty}^{\infty} e^{-t n^{2}}=\sqrt{\frac{t}{\pi}} \theta_{3}\left(0, e^{-t}\right) .
$$

This enables us to express the function $\Theta_{j}$ in terms of the function $\Omega$ as follows

$$
\Theta_{j}(t)=\frac{\sqrt{\pi}}{4} t^{-3 / 2} e^{t[j(j+1)+1]} \sum_{|\mu| \leq j} e^{-\mu^{2} t}\left[\left(1-2 \mu^{2} t\right) \Omega(t)-2 t \Omega^{\prime}(t)\right] .
$$

By using the duality relation, the function $\Theta_{j}$ takes the form

$$
\Theta_{j}(t)=e^{t[j(j+1)+1]} \sum_{n=-\infty}^{\infty} \sum_{|\mu| \leq j} \frac{1}{2}\left(n^{2}-\mu^{2}\right) e^{-t\left(n^{2}+\mu^{2}\right)} .
$$

Finally by using the obvious equation

$$
\sum_{\mu=-j}^{j} \sum_{n=-j}^{j}\left(n^{2}-\mu^{2}\right) e^{-t\left(n^{2}+\mu^{2}\right)}=0
$$

we get the heat trace of pure Laplacian in the irreducible representation $j$

$$
\begin{aligned}
\Theta_{j}(t)=\sum_{n=j+1}^{\infty}\{ & n^{2} \exp \left\{-t\left[n^{2}-j(j+1)-1\right]\right\} \\
& \left.+\sum_{\mu=1}^{j} 2\left(n^{2}-\mu^{2}\right) \exp \left\{-t\left[n^{2}+\mu^{2}-j(j+1)-1\right]\right\}\right\} .
\end{aligned}
$$

In particular, the eq. (4.29) gives the eigenvalues and their multiplicities of the pure Laplacian acting on an irreducible representation $j$ of $\mathrm{SU}(2)$. It is labeled by two integers $n$ and $\mu$ such that

$$
0 \leq \mu \leq j<n
$$

The eigenvalues are given by

$$
\lambda_{n, \mu}\left(-\Delta_{j}\right)=n^{2}+\mu^{2}-j(j+1)-1,
$$

and their multiplicities are

$$
d_{n, 0}\left(-\Delta_{j}\right)=n^{2}
$$


for $\mu=0$ and

$$
d_{n, \mu}\left(-\Delta_{j}\right)=2\left(n^{2}-\mu^{2}\right)
$$

for $1 \leq \mu \leq j$.

The minimal eigenvalue of the Laplacian $-\Delta_{j}$ is

$$
\lambda_{\min }\left(-\Delta_{j}\right)=j
$$

with multiplicity $d_{\min }\left(-\Delta_{j}\right)=(j+1)^{2}$. In particular, this means that all Laplacians $-\Delta_{j}$ for $j \geq 1$ are positive and the scalar Laplacian $\Delta_{0}$ is non-negative, it has the obvious constant zero mode.

We will need the functions $\Theta_{0}, \Theta_{1}$ and $\Theta_{2}$,

$$
\begin{aligned}
& \Theta_{0}(t)=\frac{\sqrt{\pi}}{4} t^{-3 / 2} e^{t}\left[\Omega(t)-2 t \Omega^{\prime}(t)\right] \\
&=\sum_{n=1}^{\infty} n^{2} e^{-t\left(n^{2}-1\right)}, \\
& \Theta_{1}(t)=\frac{\sqrt{\pi}}{4} t^{-3 / 2}\left\{\left[e^{3 t}+2(1-2 t) e^{2 t}\right] \Omega(t)-2 t\left[e^{3 t}+2 e^{2 t}\right] \Omega^{\prime}(t)\right\} \\
&=\sum_{n=2}^{\infty}\left\{n^{2} e^{-t\left(n^{2}-3\right)}+2\left(n^{2}-1\right) e^{-t\left(n^{2}-2\right)}\right\}, \\
& \Theta_{2}(t)=\frac{\sqrt{\pi}}{4} t^{-3 / 2}\left\{\left[e^{7 t}+2(1-2 t) e^{6 t}+2(1-8 t) e^{3 t}\right] \Omega(t)\right. \\
&\left.\quad-2 t\left[e^{7 t}+2 e^{6 t}+2 e^{3 t}\right] \Omega^{\prime}(t)\right\}
\end{aligned}
$$

It is worth noting that the contribution of $\mu=0$ and $\mu=1$ for $j=1$ corresponds to the decomposition of the vector fields

$$
\varphi_{\mu}=A_{\mu}^{\perp}+\nabla_{\mu} \sigma
$$

where $A_{\mu}$ is the transversal (divergence free) vector, and the contribution of $\mu=0, \mu=$ 1 and $\mu=2$ for $j=2$ corresponds to the decomposition of the trace-free symmetric tensor fields

$$
\varphi_{\mu \nu}=\varphi_{\mu \nu}^{\perp}+2 \nabla_{(\mu} A_{\nu)}^{\perp}+\nabla_{\mu} \nabla_{\nu} \sigma-\frac{1}{3} g_{\mu \nu} \Delta \sigma
$$

where $\varphi_{\mu \nu}^{\perp}$ is the transversal (divergence free) tracefree tensor and $\sigma$ is a scalar.

\subsection{Heat trace of quantum gravity}

We introduce the trace-free tensor part and the scalar part of the graviton operator $L_{2}$

$$
\begin{aligned}
& L_{2}^{(0)}=\Pi_{0} L_{2} \Pi_{0}, \\
& L_{2}^{(2)}=\Pi_{2} L_{2} \Pi_{2} .
\end{aligned}
$$


Now, by using the eqs. (4.18) and (4.21) we compute the eigenvalues of the operators $L_{1}$ and $L_{2}$

$$
\begin{array}{clrl}
\lambda_{n, \mu}\left(L_{1}\right)=\lambda_{n, \mu}\left(-\Delta_{1}\right)-2=n^{2}+\mu^{2}-5, & n \geq 2, & \mu=0,1, \\
\lambda_{n, \mu}\left(L_{2}^{(0)}\right)=\lambda_{n, 0}\left(-\Delta_{0}\right)-2-2 \lambda=n^{2}-3-2 \lambda, & n \geq 1, & \\
\lambda_{n, \mu}\left(L_{2}^{(2)}\right)=\lambda_{n, \mu}\left(-\Delta_{2}\right)+4-2 \lambda=n^{2}+\mu^{2}-3-2 \lambda, & n \geq 3, & \mu=0,1,2 .
\end{array}
$$

The minimal eigenvalues are

$$
\begin{aligned}
\lambda_{\min }\left(L_{1}\right) & =-1, \\
\lambda_{\min }\left(L_{2}^{(0)}\right) & =-2-2 \lambda, \\
\lambda_{\min }\left(L_{2}^{(2)}\right) & =6-2 \lambda .
\end{aligned}
$$

Notice that the minimal eigenvalue of the ghost operator $L_{1}$ is always negative, the minimal eigenvalue of the conformal sector of the graviton operator $L_{2}^{(0)}$ is negative for $\lambda>-1$ and the minimal eigenvalue of the graviton operator in the traceless tensor part $L_{2}^{(2)}$ is negative for $\lambda>3$. That is, the graviton operator is positive only for negative cosmological constant when $\lambda<-1$.

Next, by using eq. (2.28) and (4.21) we get

$$
\Theta_{\mathrm{GR}}(t)=e^{(-4+2 \lambda) t} \Theta_{2}(t)+e^{(2+2 \lambda) t} \Theta_{0}(t)-2 e^{2 t} \Theta_{1}(t) .
$$

We can write this either in terms of the function $\Omega$

$$
\begin{aligned}
\Theta_{\mathrm{GR}}(t)=\frac{\sqrt{\pi}}{4} t^{-3 / 2}\{ & -2 t\left[e^{2 \lambda t}\left(2 e^{3 t}+2 e^{2 t}+2 e^{-t}\right)-2 e^{5 t}-4 e^{4 t}\right] \Omega^{\prime}(t) \\
& \left.+\left[e^{2 \lambda t}\left(2 e^{3 t}+2(1-2 t) e^{2 t}+2(1-8 t) e^{-t}\right)-2 e^{5 t}-4(1-2 t) e^{4 t}\right] \Omega(t)\right\},
\end{aligned}
$$

which is useful in the ultraviolet limit as $t \rightarrow 0$, or in the spectral form

$$
\begin{aligned}
& \Theta_{\mathrm{GR}}(t)= e^{(2+2 \lambda) t}+4 e^{(-1+2 \lambda) t}-8 e^{t}-12 \\
&+\sum_{n=3}^{\infty}\left\{e^{2 \lambda t}\left\{2 n^{2} e^{-t\left(n^{2}-3\right)}+2\left(n^{2}-1\right) e^{-t\left(n^{2}-2\right)}+2\left(n^{2}-4\right) e^{-t\left(n^{2}+1\right)}\right\}\right. \\
&\left.\quad-2 n^{2} e^{-t\left(n^{2}-5\right)}-4\left(n^{2}-1\right) e^{-t\left(n^{2}-4\right)}\right\}
\end{aligned}
$$

which is useful in the infrared limit as $t \rightarrow \infty$.

When the classical Einstein equations are satisfied, that is, when $\lambda=1$, the heat trace simplifies to

$$
\begin{aligned}
\Theta_{\mathrm{GR}}(t) & =\frac{\sqrt{\pi}}{4} t^{-3 / 2}\left\{\left[-2(1-2 t) e^{4 t}+2(1-8 t) e^{t}\right] \Omega(t)-2 t\left[-2 e^{4 t}+2 e^{t}\right] \Omega^{\prime}(t)\right\} \\
& =e^{4 t}-4 e^{t}-12+\sum_{n=3}^{\infty}\left\{-2\left(n^{2}-1\right) e^{-t\left(n^{2}-4\right)}+2\left(n^{2}-4\right) e^{-t\left(n^{2}-1\right)}\right\} .
\end{aligned}
$$


The infrared properties are described by the limit $t \rightarrow \infty$. By using the spectral representation of the heat trace we immediately get

$$
\Theta_{\mathrm{GR}}(t)=e^{(2+2 \lambda) t}+4 e^{(-1+2 \lambda) t}-8 e^{t}-12+O\left(e^{(2 \lambda-6) t}\right) .
$$

The exponential growth of the heat trace indicates the presence of the negative modes.

It is instructive to study the asymptotics of the heat trace as $t \rightarrow 0$. By using the asymptotics of the function $\Omega$ as $t \rightarrow 0$

$$
\Omega(t) \sim 1, \quad \Omega^{\prime}(t) \sim 0
$$

we obtain

$$
\Theta_{\mathrm{GR}}(t)=\frac{\sqrt{\pi}}{4} t^{-3 / 2}\left\{C_{0}+t C_{1}+t^{2} C_{2}+O\left(t^{3}\right)\right\}
$$

where

$$
\begin{aligned}
& C_{0}=0, \\
& C_{1}=2 \pi^{2}(12 \lambda-30), \\
& C_{2}=2 \pi^{2}\left(12 \lambda^{2}-24 \lambda-3\right) .
\end{aligned}
$$

These coefficients coincide with the coefficients $C_{k}$ given by the general formulas (3.61)(3.63) in three dimensions, $n=3$. Notice the absence of the constant term here. This is the feature of three-dimensional quantum Einstein gravity since it does not have any dynamics, that is, the number of degrees of freedom is equal to zero.

\section{$5 \quad$ Heat traces on $S^{1} \times S^{3}$}

\subsection{Reduction of heat traces}

In this section we study Einstein quantum gravity in the physical four-dimensional Einstein Universe. Since we would like to study the thermal effects at the same time, we consider the four-dimensional Riemannian manifold $M=S^{1} \times S^{3}$ with a circle $S^{1}$ of radius $a_{1}$ and a sphere $S^{3}$ of radius $a$. So, all indices in this section are four-dimensional, that is, they run over $1,2,3,4$.

Let $h_{b}^{a}$ be the projection tensor on $S^{3}$ and $q^{a}{ }_{b}$ be the projection to $S^{1}$ so that

$$
\delta_{b}^{a}=q_{b}^{a}+h_{b}^{a},
$$

and

$$
\begin{aligned}
& h^{a}{ }_{b} h^{b}{ }_{c}=h^{a}{ }_{c} \text {, } \\
& q^{a}{ }_{b} q^{b}{ }_{c}=q^{a}{ }_{c}, \\
& h^{a}{ }_{b} q^{b}=0, \\
& h^{a}{ }_{a}=3 \text {, } \\
& q^{a}{ }_{a}=1 .
\end{aligned}
$$

Also, we introduce the Levi-Civita tensor $\varepsilon_{a b c}$ on $S^{3}$ such that

$$
\varepsilon_{a b c} q_{d}^{a}=0
$$


and

$$
\begin{aligned}
& \varepsilon_{a b c} \varepsilon^{d e f}=6 h_{[a}^{d} h_{b}^{e} h_{c]}^{f}, \\
& \varepsilon_{a b c} \varepsilon^{d e c}=2 h^{d}{ }_{[a}^{e} h_{b]}^{e}, \\
& \varepsilon_{a b c} \varepsilon^{d b c}=2 h^{d}{ }_{a} .
\end{aligned}
$$

Then the curvature is

$$
\begin{aligned}
R_{c d}^{a b} & =\frac{1}{a^{2}}\left(h^{a}{ }_{c} h_{d}^{b}-h_{d}^{a} h_{c}^{b}\right), \\
R_{a b} & =\frac{2}{a^{2}} h_{a b}, \\
R & =\frac{6}{a^{2}} .
\end{aligned}
$$

The volume of the manifold $M=S^{1} \times S^{3}$ is

$$
\operatorname{vol}(M)=4 \pi^{3} a_{1} a^{3},
$$

and the classical action is equal to

$$
S=\frac{\pi^{2}}{2 G} a_{1} a\left(-3+a^{2} \Lambda\right) .
$$

Thus, the potential terms (2.35), (2.36), of the operators $L_{1}$ and $L_{2}$ are

$$
\begin{aligned}
a^{2}\left(Q_{1}\right)_{b}^{a}= & -2 h_{b}^{a}, \\
a^{2}\left(Q_{2}\right)^{a b}{ }_{c d}= & (6-2 \lambda) \delta^{a}{ }_{\left({ }_{c} \delta^{b}{ }_{d)}-2 h^{(a}{ }_{\left({ }_{c} h^{b)} d\right)}-h^{a b} h_{c d}\right.} \\
& -h^{a b} q_{c d}-q^{a b} h_{c d}-4 q^{(a}{ }_{(c} h^{b)}{ }_{d)}-3 q^{a b} q_{c d} .
\end{aligned}
$$

We need to compute the heat traces of the Laplace type operators $L_{j}=-\Delta+Q_{j}(2.34)$ on $M=S^{1} \times S^{3}$. We note that since the potential terms are constant we have

$$
\exp \left(-t L_{j}\right)=\exp \left(-t Q_{j}\right) \exp (t \Delta)
$$

and also

$$
\exp \left(t \Delta^{S^{1} \times S^{3}}\right)=\exp \left(t \Delta^{S^{1}}\right) \exp \left(t \Delta^{S^{3}}\right) .
$$

Therefore, the heat traces $\Theta_{L_{j}}(t)$ of the operators $L_{j}$ can be computed as follows

$$
\Theta_{L_{j}}(t)=\Theta^{S^{1}}\left(\frac{t}{a_{1}^{2}}\right) \Theta_{L_{j}}^{S^{3}}\left(\frac{t}{a^{2}}\right)
$$

where

$$
\Theta^{S^{1}}(t)=\sqrt{\frac{\pi}{t}} \Omega(t)
$$

with $\Omega(t)$ defined by (4.23), is the heat trace on the unit circle $S^{1}$ and

$$
\Theta_{L_{j}}^{S^{3}}(t)=\operatorname{Tr} \exp \left(-t L_{j}^{S^{3}}\right)
$$

is the heat trace on the unit sphere $S^{3}$. 
The heat trace $\Theta_{L_{j}}^{S^{3}}(t)$ on $S^{3}$ was computed in our paper [12]. We consider a tensor representation of spin $j$ of the spin group $\operatorname{Spin}(4)$ with generators $\Sigma_{(j)}^{a b}$ satisfying the algebra (3.5). Recall that $\operatorname{Spin}(4)=\mathrm{SU}(2) \times \mathrm{SU}(2)$. Therefore, the matrices

$$
G_{(j) i}=\frac{1}{2} \varepsilon_{i a b} \Sigma_{(j)}^{a b},
$$

satisfy the algebra (no summation over $j !$ )

$$
\left[G_{(j) i}, G_{(j) k}\right]=-\varepsilon^{l}{ }_{i k} G_{(j) l}
$$

and form a reducible representation of the group $\mathrm{SU}(2)$; with the Casimir operator (no summation over $j !$ )

$$
G_{(j)}^{2}=G_{(j) i} G_{(j) i}
$$

We also define the matrix

$$
G_{(j)}(y)=G_{(j) i} y^{i},
$$

where $y=\left(y^{i}\right)$ is a unit vector. Let $f$ be a real-valued function of $x=\left(x^{i}\right) \in \mathbb{R}^{3}$. Let $x^{i}=r y^{i}$, where $r=|x|=\sqrt{x^{i} x_{i}}$ and $y=\left(y^{i}\right)$ is the unit vector such that $|y|=1$. Of course, the unit vector $y$ lies on the unit sphere $S^{2}$ in $\mathbb{R}^{3}$. We introduce the average over the unit sphere $S^{2}$ of functions in $\mathbb{R}^{3}$ by

$$
\langle f\rangle(r)=\frac{1}{4 \pi} \int_{S^{2}} d y_{S^{2}} f(r y) ;
$$

the integration goes over the unit sphere $S^{2}$ with the appropriate induced metric on $S^{2}$.

Then the heat trace of the Laplace type operator $L_{j}=-\Delta+Q_{j}$ on the unit sphere $S^{3}$ has the form [12]

$$
\Theta_{L_{j}}^{S^{3}}(t)=\frac{\sqrt{\pi}}{4} t^{-3 / 2} \operatorname{tr} \exp \left[-t\left(G_{(j)}^{2}+Q_{j}-I_{j}\right)\right] S_{j}(t),
$$

where $S_{j}(t)$

$$
S_{j}(t)=\sum_{n=-\infty}^{\infty} \exp \left(-\frac{\pi^{2} n^{2}}{t}\right) \int_{-\infty}^{\infty} \frac{d r}{\sqrt{\pi}} e^{-r^{2}}\left(2 r^{2}-2 \frac{\pi^{2} n^{2}}{t}\right)\left\langle\exp \left[2 r \sqrt{t} G_{(j)}(y)\right]\right\rangle .
$$

\subsection{Generators}

For the vector and the symmetric 2-tensor representation (3.7) and (3.16) the generators have the form

$$
\begin{aligned}
\left(G_{(1) i}\right)^{c} d & =\varepsilon_{i}^{c}{ }_{d}, \\
\left(G_{(2) i}\right)^{e f}{ }_{c d} & \left.=2 \varepsilon_{i}^{(e}{ }_{(c} \delta^{f)} d\right),
\end{aligned}
$$

so that

$$
\begin{aligned}
\left(G_{(1)}(y)\right)^{a}{ }_{b} & =\varepsilon_{i}{ }^{a}{ }_{b} y^{i}, \\
\left(G_{(2)}(y)\right)^{a b}{ }_{c d} & =2 \varepsilon_{i}{ }^{(a}{ }_{(c} \delta^{b)}{ }_{d)} y^{i} .
\end{aligned}
$$


We compute the Casimir operators

$$
\begin{aligned}
\left(G_{(1)}^{2}\right)^{c}{ }_{d} & =-2 h_{d}^{c}, \\
\left(G_{(2)}^{2}\right)^{e f}{ }_{c d} & =-6 h^{(e}{ }_{(c} h^{f)}{ }_{d)}+2 h^{e f} h_{c d}-4 q^{(e}{ }_{(c} h^{f)}{ }_{d)}
\end{aligned}
$$

and the sums

$$
\begin{aligned}
\left(G_{(1)}^{2}+Q_{1}\right)^{c}{ }_{d}= & -4 h_{d}^{c}, \\
\left(G_{(2)}^{2}+Q_{2}\right)^{a b}{ }_{c d}= & (6-2 \lambda) \delta^{a}{ }_{\left({ }_{c} \delta^{b}{ }_{d)}-8 h^{(a}{ }_{\left({ }_{c}\right.} h^{(b)}{ }_{d)}+h^{a b} h_{c d}\right.} \\
& -h^{a b} q_{c d}-q^{a b} h_{c d}-8 q^{(a}{ }_{(c} h^{(b)}{ }_{d)}-3 q^{a b} q_{c d} .
\end{aligned}
$$

\subsection{Algebra of constant symmetric endomorphisms}

First of all, for the vector representation we immediately obtain

\section{Lemma 1}

$$
\exp \left\{-t\left(G_{(1)}^{2}+Q_{1}\right)\right\}=I_{1}-H+e^{4 t} H,
$$

where $H$ is the matrix of the projection $H=\left(h^{a}{ }_{b}\right)$.

To compute this exponential for the tensor representation we need to do some algebra. We define the following basis of endomorphisms acting on symmetric two-tensors in four dimensions

$$
\begin{aligned}
I^{a b}{ }_{c d} & =\delta^{(a}{ }_{(c} \delta^{b)}{ }_{d)}, \\
A^{a b}{ }_{c d} & =h^{(a}{ }_{\left({ } h^{b)} d\right)}, \\
B^{a b}{ }_{c d} & =h^{a b} h_{c d}, \\
C^{a b}{ }_{c d} & =h^{a b} q_{c d}, \\
D^{a b}{ }_{c d} & =q^{a b} h_{c d}, \\
E^{a b}{ }_{c d} & =q^{(a}{ }_{(c} h^{b)}{ }_{d)}, \\
F^{a b}{ }_{c d} & =q^{a b} q_{c d} .
\end{aligned}
$$

First, we note the identity

$$
A+2 E+F=I,
$$

so that

$$
E=\frac{1}{2}(I-A-F) .
$$

Of course, $I$ is the identity. We compute the squares of these endomorphisms

$$
\begin{aligned}
& A^{2}=A, \\
& B^{2}=3 B, \\
& C^{2}=0, \\
& D^{2}=0, \\
& E^{2}=\frac{1}{2} E, \\
& F^{2}=F,
\end{aligned}
$$


and their products

$$
\begin{aligned}
& A B=B, \quad B A=B, \\
& A C=C, \quad C A=0 \text {, } \\
& A D=0, \quad D A=D, \\
& A E=0, \quad E A=0 \text {, } \\
& A F=0, \quad F A=0, \\
& B C=3 C, \quad C B=0, \\
& B D=0, \quad D B=3 D, \\
& B E=0, \quad E B=0 \text {, } \\
& B F=0, \quad F B=0, \\
& C D=B, \quad D C=3 F, \\
& C E=0, \quad E C=0, \\
& C F=C, \quad F C=0, \\
& D E=0, \quad E D=0 \text {, } \\
& D F=0, \quad F D=D \\
& E F=0, \quad F E=0 \text {. }
\end{aligned}
$$

Next, we define the following endomorphisms

$$
\begin{aligned}
P_{1} & =A-\frac{1}{3} B, \\
P_{2} & =\frac{1}{3} B, \\
P_{3} & =2 E, \\
P_{4} & =F \\
T & =C+D, \\
X & =\frac{1}{2}\left(P_{4}-P_{2}-T\right), \\
\Pi_{ \pm} & =\frac{1}{2}\left(P_{2}+P_{4} \pm X\right) .
\end{aligned}
$$

By using the algebra of these endomorphisms one can prove

Lemma 2 The endomorphisms $P_{1}, P_{2}, P_{3}$ and $P_{4}$ form a set of orthogonal projections satisfying

$$
\begin{aligned}
P_{i}^{2} & =P_{i}, \\
P_{i} P_{j} & =0, \quad \text { if } \quad i \neq j,
\end{aligned}
$$

and

$$
P_{1}+P_{2}+P_{3}+P_{4}=I \text {. }
$$


The dimensions of the corresponding subspaces are determined by the traces

$$
\operatorname{tr} P_{1}=5, \quad \operatorname{tr} P_{2}=1, \quad \operatorname{tr} P_{3}=3, \quad \operatorname{tr} P_{4}=1 .
$$

Of course, the total dimension of the space of symmetric two-tensors in four dimensions is

$$
5+1+3+1=10 .
$$

Lemma 3 1. The endomorphism $X$ satisfies the equations

$$
\begin{aligned}
X P_{1} & =P_{1} X=P_{3} X=X P_{3}=0 . \\
\left(P_{2}+P_{4}\right) X & =X\left(P_{2}+P_{4}\right)=X . \\
X^{2} & =P_{2}+P_{4} . \\
\operatorname{tr} X & =0
\end{aligned}
$$

It has the eigenvalue 0 with multiplicity 8 and simple eigenvalues $-1,+1$.

2. The endomorphisms $\Pi_{ \pm}$are the projections to the eigenspaces of $X$ corresponding to the eigenvalues \pm 1 . They satisfy the equations

$$
\begin{aligned}
\Pi_{ \pm}^{2} & =\Pi_{ \pm}, \\
\Pi_{-} \Pi_{+} & =\Pi_{+} \Pi_{-}=0, \\
X \Pi_{ \pm} & = \pm \Pi_{ \pm}, \\
\Pi_{+}\left(P_{2}+P_{4}\right) & =\Pi_{+}, \\
\operatorname{tr} \Pi_{ \pm} & =1,
\end{aligned}
$$

Proof. The projections $P_{i}$ act on the matrices $C$ and $D$ by

$$
\begin{array}{ll}
P_{1} C=0, & C P_{1}=0, \\
P_{2} C=C, & C P_{2}=0, \\
P_{3} C=0, & C P_{3}=0, \\
P_{4} C=0, & C P_{4}=C, \\
P_{1} D=0, & D P_{1}=0, \\
P_{2} D=0, & D P_{2}=D, \\
P_{3} D=0, & D P_{3}=0, \\
P_{4} D=D, & D P_{4}=0 .
\end{array}
$$

and, therefore,

$$
\begin{array}{ll}
P_{1} T=0, & T P_{1}=0, \\
P_{2} T=C, & T P_{2}=D, \\
P_{3} T=0, & T P_{3}=0, \\
P_{4} T=D, & T P_{4}=C,
\end{array}
$$


so that

$$
P_{2} T+T P_{2}=T, \quad P_{4} T+T P_{4}=T .
$$

Also, we have

$$
T^{2}=3\left(P_{2}+P_{4}\right) .
$$

By using these equations one can prove all the equations of the lemma.

Since the matrix $X$ is orthogonal to the projections $P_{1}$ and $P_{3}$, it has an obvious eigenvalue equal to zero with multiplicity $8=5+3$ making it essentially two-dimensional. It acts nontrivially only on subspaces spanned by projections $P_{2}$ and $P_{4}$, which are both one-dimensional. Since it is obviously traceless, the sum of its eigenvalues is equal to zero. It is easy to see that it has two non-zero eigenvalues \pm 1 . This follows from the eqs. (5.80).

The matrices $\Pi_{ \pm}$are the eigenprojections corresponding to the eigenvalues \pm 1 ; this follows from the eqs. (5.84).

We prove the following

\section{Lemma 4}

$$
\exp \left\{-t\left[G_{(2)}^{2}+Q_{2}\right]\right\}=e^{2 \lambda t}\left\{P_{1} e^{2 t}+P_{3} e^{-2 t}+\Pi_{-}+\Pi_{+} e^{-4 t}\right\} .
$$

Proof. We have

$$
G_{(2)}^{2}+Q_{2}=(2-2 \lambda) I-4 P_{1}+2 X,
$$

Therefore,

$$
\exp \left\{-t\left[G_{(2)}^{2}+Q_{2}\right]\right\}=\exp \{-t(2-2 \lambda)\} \exp \left(4 t P_{1}\right) \exp (-2 t X)
$$

We compute

$$
\exp \left(4 t P_{1}\right)=P_{1} e^{4 t}+P_{2}+P_{3}+P_{4} .
$$

The only thing left to compute is the exponential $\exp (-2 t X)$. By using

$$
X^{2 n}=P_{2}+P_{4}, \quad X^{2 n+1}=X,
$$

we get

$$
\exp (-2 t X)=P_{1}+P_{3}+\Pi_{-} e^{2 t}+\Pi_{+} e^{-2 t} .
$$

This finally gives the eq. (5.101).

\subsection{Algebra of symmetric endomorphisms on $S^{3}$}

Let $y=\left(y^{i}\right)$ be a unit vector orthogonal to $q_{b}^{a}$, that is, satisfying $y^{a}=h^{a}{ }_{b} y^{b}$. We introduce two matrices

$$
Z_{b}^{a}=y^{i} \varepsilon_{i}^{a}{ }_{b},
$$

and

$$
P_{b}^{a}=h_{b}^{a}-y^{a} y_{b}
$$


The square of the matrix $Z$ is equal to

$$
Z^{2}=-P
$$

and the matrix $P$ is obviously a projection so that

$$
\begin{aligned}
P^{2} & =P, \\
P Z & =Z P=Z, \\
P H & =H P=P,
\end{aligned}
$$

and

$$
\operatorname{tr} Z=0, \quad \operatorname{tr} P=2, \quad \operatorname{tr} H=3 .
$$

First, we prove

Lemma 5 The exponential of the matrix $G_{(1)}(y)$ is

$$
\exp \left[2 r G_{(1)}(y)\right]=\cos (2 r) P+\sin (2 r) Z
$$

with the trace

$$
\operatorname{tr} \exp \left(2 r G_{(1)}(y)\right)=2 \cos (2 r) .
$$

Proof. This follows from the fact that

$$
G_{(1)}(y)=Z
$$

and the eq. (5.109).

Next, we introduce the following endomorphisms acting on symmetric two-tensors

$$
\begin{aligned}
K^{a b}{ }_{c d} & \left.=Z^{(a}{ }_{(c} \delta^{b)} d\right), \\
L^{a b}{ }_{c d} & =Z^{(a}{ }_{\left({ }_{c} Z^{b)} d\right)}, \\
W^{a b}{ }_{c d} & \left.=Z^{(a}{ }_{(c} P^{b)} d\right), \\
M^{a b}{ }_{c d} & =P^{(a}{ }_{\left({ }_{c} \delta^{b)} d\right)}, \\
N^{a b}{ }_{c d} & =P^{(a}{ }_{\left({ }{ }^{b}{ }^{b)} d\right)}, \\
S^{a b}{ }_{c d} & =P^{a b} P_{c d}, \\
U^{a b}{ }_{c d} & =P^{a b} g_{c d}, \\
Y^{a b}{ }_{c d} & =g^{a b} P_{c d} .
\end{aligned}
$$

We compute the traces

$$
\begin{aligned}
\operatorname{tr} K & =0, \\
\operatorname{tr} L & =-1, \\
\operatorname{tr} M & =5, \\
\operatorname{tr} N & =3, \\
\operatorname{tr} W & =0 .
\end{aligned}
$$


We need to compute the algebra of these endomorphisms. First, we have

$$
\begin{aligned}
K^{2} & =-\frac{1}{2} M+\frac{1}{2} L, \\
M^{2} & =M, \\
L^{2} & =N, \\
N^{2} & =N, \\
L M & =L, \\
M L & =L, \\
K M & =\frac{1}{2}(K+W), \\
K L & =-W, \\
N M & =N, \\
N L & =L, \\
K N & =W, \\
K W & =\frac{1}{2}(-N+L) .
\end{aligned}
$$

We prove the following

Lemma 6 The exponential of the endomorphism $G_{(2)}(y)$ has the form

$$
\exp \left[2 r G_{(2)}(y)\right]=\gamma(r) I+\mu(r) M+\nu(r) N+\lambda(r) L+\eta(r) W+\varkappa(r) K
$$

where

$$
\begin{aligned}
\gamma(r) & =1, \\
\mu(r) & =2 \cos (2 r)-2, \\
\nu(r) & =\frac{1}{2} \cos (4 r)-2 \cos (2 r)+\frac{3}{2}, \\
\lambda(r) & =\frac{1}{2}-\frac{1}{2} \cos (4 r), \\
\eta(r) & =\sin (4 r)-2 \sin (2 r), \\
\varkappa(r) & =2 \sin (2 r) .
\end{aligned}
$$

with the trace

$$
\operatorname{tr} \exp \left[2 r G_{(2)}(y)\right]=4+4 \cos (2 r)+2 \cos (4 r) .
$$

Proof. We note that

$$
G_{(2)}(y)=2 K
$$

Let

$$
J(r)=\exp (4 r K)
$$


It satisfies the differential equation

$$
\partial_{r} J=4 K J
$$

with initial condition

$$
J(0)=I
$$

We decompose it according to

$$
J=\gamma I+\mu M+\nu N+\lambda L+\eta W+\varkappa K .
$$

Then by using the algebra of the matrices $M, N, L, W, K$ we have

$$
K J=-\frac{\varkappa}{2} M-\frac{\eta}{2} N+\frac{1}{2}(\eta+\varkappa) L+\left(\frac{\mu}{2}+\nu-\lambda\right) W+\left(\frac{\mu}{2}+\gamma\right) K .
$$

Therefore, the coefficients of this expansion must satisfy the differential equations

$$
\begin{aligned}
& \partial_{r} \gamma=0, \\
& \partial_{r} \mu=-2 \varkappa, \\
& \partial_{r} \nu=-2 \eta, \\
& \partial_{r} \lambda=2 \eta+2 \varkappa, \\
& \partial_{r} \eta=2 \mu+4 \nu-4 \lambda, \\
& \partial_{r} \varkappa=2 \mu+4 \gamma,
\end{aligned}
$$

with the initial conditions

$$
\gamma(0)=1, \quad \mu(0)=\nu(0)=\lambda(0)=\eta(0)=\varkappa(0)=0 .
$$

The solution of this system gives the result (5.143)-(5.148). Now the trace can be easily computed.

\subsection{Group averages}

Next, we need to compute the group averages (5.24) of the functions given by (5.114) and (5.142). Thus, we need to compute the averages of the polynomials. We prove

Lemma 7 The averages of the monomials are

$$
\begin{aligned}
\langle 1\rangle & =1 \\
\left\langle y^{i_{1}} \cdots y^{i_{2 k+1}}\right\rangle & =0 \\
\left\langle y^{i_{1}} \cdots y^{i_{2 k}}\right\rangle & =\frac{1}{2 k+1} \delta^{\left(i_{1} i_{2}\right.} \cdots \delta^{\left.i_{2 k-1} i_{2 k}\right)} .
\end{aligned}
$$

Proof. The first two equations are obvious. To prove the eq. (5.165) we consider the Gaussian integral

$$
\int_{\mathbb{R}^{3}} d x e^{-|x|^{2}} x^{i_{1}} \cdots x^{i_{2 n}}=\pi^{3 / 2} \frac{(2 n) !}{n ! 2^{2 n}} \delta^{\left(i_{1} i_{2}\right.} \cdots \delta^{\left.i_{2 n-1} i_{2 n}\right)} .
$$


By changing the variables here by $x^{i}=r y^{i}$ and using the integral

$$
\int_{0}^{\infty} d r r^{2 n+2} e^{-r^{2}}=\sqrt{\pi} \frac{(2 n+2) !}{(n+1) ! 2^{2 n+3}}
$$

we get eq. (5.165).

Corollary 1 Let $h$ be the projection onto the three-dimensional subspace $V=\mathbb{R}^{3}$ of $\mathbb{R}^{4}$ and $y=\left(y^{i}\right)$ be a four-dimensional unit vector lying in $V$. Then the eq. (5.165) is modified as follows

$$
\left\langle y^{a_{1}} \cdots y^{a_{2 k}}\right\rangle=\frac{1}{2 k+1} h^{\left(a_{1} i_{2}\right.} \cdots h^{\left.a_{2 k-1} a_{2 k}\right)} .
$$

We define the characters of an irreducible representation $j$ of $\mathrm{SU}(2)$ by

$$
\chi_{j}(r)=\operatorname{tr}_{j}\left\langle\exp \left[2 r G_{(j)}(y)\right]\right\rangle,
$$

where $\operatorname{tr}_{j}$ is the trace in the irreducible representation $j$. For an irreducible representation $j$ the average of a group element over the $S^{2}$ is given by eq. (5.55) of [12]:

$$
\left\langle\exp \left[2 r G_{(j)}(y)\right]\right\rangle=\frac{1}{2 j+1} \sum_{|\mu| \leq j} \cos (2 \mu r) \Pi_{j},
$$

so that

$$
\chi_{j}(r)=\sum_{|\mu| \leq j} \cos (2 \mu r)
$$

in particular,

$$
\begin{aligned}
& \chi_{1}(r)=1+2 \cos (2 r), \\
& \chi_{2}(r)=1+2 \cos (2 r)+2 \cos (4 r) .
\end{aligned}
$$

Using the averages of the monomials calculated above we obtain

$$
\begin{aligned}
\langle Z\rangle & =0 \\
\langle P\rangle & ==\frac{2}{3} h, \\
\langle M\rangle & =\frac{2}{3}\left(P_{1}+P_{2}+\frac{1}{2} P_{3}\right), \\
\langle N\rangle & =\frac{7}{15} P_{1}+\frac{2}{3} P_{2}, \\
\langle L\rangle & =\frac{1}{3}\left(2 P_{2}-P_{1}\right) \\
\langle W\rangle & =0 \\
\langle K\rangle & =0
\end{aligned}
$$


This allows us to calculate the group averages of the exponentials

$$
\begin{aligned}
\left\langle\exp \left[2 r G_{(1)}(y)\right]\right\rangle= & \frac{2}{3} H \cos (2 r) \\
\left\langle\exp \left[2 r G_{(2)}(y)\right]\right\rangle= & \frac{1}{5}[1+2 \cos (2 r)+2 \cos (4 r)] P_{1}+P_{2}+P_{4} \\
& +\frac{1}{3}[1+2 \cos (2 r)] P_{3}
\end{aligned}
$$

\subsection{Heat trace of operator $L_{1}$ on $S^{3}$}

To compute the functions $S_{j}$ we will need the integrals

$$
\begin{aligned}
\int_{-\infty}^{\infty} d r e^{-r^{2}} \cos (2 \mu \sqrt{t} r) & =\sqrt{\pi} e^{-t \mu^{2}} \\
\int_{-\infty}^{\infty} d r e^{-r^{2}} \cos (2 \mu \sqrt{t} r) r^{2} & =\sqrt{\pi}\left(\frac{1}{2}-\mu^{2} t\right) e^{-t \mu^{2}}
\end{aligned}
$$

By using these integrals we obtain

$$
\int_{-\infty}^{\infty} d r e^{-r^{2}}\left(2 r^{2}-\frac{2 \pi^{2} n^{2}}{t}\right) \cos (2 r \sqrt{t})=\sqrt{\pi}\left(1-2 t-\frac{2 \pi^{2} n^{2}}{t}\right) e^{-t}
$$

and we finally obtain from (5.26)

$$
S_{L_{1}}(t)=\sum_{n=-\infty}^{\infty} \exp \left(-\frac{\pi^{2} n^{2}}{t}\right)\left[\left(1-\frac{2 \pi^{2} n^{2}}{t}\right)\left(I_{1}-P\right)+\left(1-2 t-\frac{2 \pi^{2} n^{2}}{t}\right) e^{-t} P\right] .
$$

The heat trace is calculated now by using (5.25) and (5.35)

$$
\begin{aligned}
\Theta_{L_{1}}^{S^{3}}(t)= & \frac{\sqrt{\pi}}{4} t^{-3 / 2} e^{t} \sum_{n=-\infty}^{\infty} \exp \left(-\frac{\pi^{2} n^{2}}{t}\right) \\
& \times \operatorname{tr}\left\{\left(1-\frac{2 \pi^{2} n^{2}}{t}\right)\left(I_{1}-H+H e^{4 t}-P e^{4 t}\right)\right. \\
& \left.+\left(1-2 t-\frac{2 \pi^{2} n^{2}}{t}\right) e^{3 t} P\right\} . \\
= & \frac{\sqrt{\pi}}{4} t^{-3 / 2} \sum_{n=-\infty}^{\infty} \exp \left(-\frac{\pi^{2} n^{2}}{t}\right) \\
& \times\left\{\left(1-\frac{2 \pi^{2} n^{2}}{t}\right)\left(e^{t}+e^{5 t}\right)+2\left(1-2 t-\frac{2 \pi^{2} n^{2}}{t}\right) e^{4 t}\right\} .
\end{aligned}
$$

The heat trace can be written in terms of the function $\Omega$,

$$
\Theta_{L_{1}}^{S^{3}}(t)=\frac{\sqrt{\pi}}{4} t^{-3 / 2}\left\{\left[e^{5 t}+e^{t}+2(1-2 t) e^{4 t}\right] \Omega(t)-2 t\left[e^{5 t}+e^{t}+2 e^{4 t}\right] \Omega^{\prime}(t)\right\} .
$$


This heat trace has the asymptotic expansion as $t \rightarrow 0$

$$
\Theta_{L_{1}}^{S^{3}}(t)=\frac{\sqrt{\pi}}{4} t^{-3 / 2}\left(4+10 t+13 t^{2}+O\left(t^{3}\right)\right)
$$

This can be put in the spectral form by using the identities

$$
\begin{aligned}
\Omega(t)-2 t \Omega^{\prime}(t) & =\frac{2 t^{3 / 2}}{\sqrt{\pi}} \sum_{n \in \mathbb{Z}} n^{2} e^{-t n^{2}}, \\
(1-2 t) \Omega(t)-2 t \Omega^{\prime}(t) & =\frac{2 t^{3 / 2}}{\sqrt{\pi}} \sum_{n \in \mathbb{Z}}\left(n^{2}-1\right) e^{-t n^{2}} .
\end{aligned}
$$

We get

$$
\Theta_{L_{1}}^{S^{3}}(t)=\frac{1}{2} \sum_{n \in \mathbb{Z}} e^{-t n^{2}}\left[n^{2}\left(e^{5 t}+e^{t}\right)+2\left(n^{2}-1\right) e^{4 t}\right] .
$$

The asymptotic behavior of the heat trace in the limit $t \rightarrow \infty$ is

$$
\Theta_{L_{1}}^{S^{3}}(t)=4 e^{t}+7+O\left(e^{-4 t}\right)
$$

\subsection{Heat trace of the operator $L_{2}$ on $S^{3}$}

Now, by using the integrals (5.183) and (5.184), we compute first

$$
\begin{aligned}
\int_{-\infty}^{\infty} d r e^{-r^{2}}\left(2 r^{2}-\frac{2 \pi^{2} n^{2}}{t}\right) & =\sqrt{\pi}\left(1-\frac{2 \pi^{2} n^{2}}{t}\right) \\
\int_{-\infty}^{\infty} d r e^{-r^{2}}\left(2 r^{2}-\frac{2 \pi^{2} n^{2}}{t}\right) \cos (4 r \sqrt{t}) & =\sqrt{\pi}\left(1-8 t-\frac{2 \pi^{2} n^{2}}{t}\right) e^{-4 t}
\end{aligned}
$$

and then by using (5.26) we find

$$
\begin{aligned}
S_{2}(t)=\sum_{n=-\infty}^{\infty} \exp \left(-\frac{\pi^{2} n^{2}}{t}\right) & {\left[\left(\frac{1}{5} P_{1}+P_{2}+\frac{1}{3} P_{3}+P_{4}\right)\left(1-\frac{2 \pi^{2} n^{2}}{t}\right)\right.} \\
+ & \left.\left(\frac{2}{5} P_{1}+\frac{2}{3} P_{3}\right)\left(1-2 t-\frac{2 \pi^{2} n^{2}}{t}\right) e^{-t}+\frac{2}{5} P_{1}\left(1-8 t-\frac{2 \pi^{2} n^{2}}{t}\right) e^{-4 t}\right] .
\end{aligned}
$$

Further, by using (5.101) and the algebra of symmetric endomorphisms we get

$$
\begin{aligned}
\exp & {\left[-t\left(G_{(2)}^{2}+Q_{2}^{2}\right)\right] S_{2}(t)=e^{2 \lambda t} \sum_{n=-\infty}^{\infty} \exp \left(-\frac{\pi^{2} n^{2}}{t}\right) } \\
& \times\left\{\left[\frac{1}{5}\left(1-\frac{2 \pi^{2} n^{2}}{t}\right) e^{2 t}+\frac{2}{5}\left(1-8 t-\frac{2 \pi^{2} n^{2}}{t}\right) e^{-2 t}+\frac{2}{5}\left(1-2 t-\frac{2 \pi^{2} n^{2}}{t}\right) e^{t}\right] P_{1}\right. \\
& \left.+\left[\frac{1}{3}\left(1-\frac{2 \pi^{2} n^{2}}{t}\right) e^{-2 t}+\frac{2}{3}\left(1-2 t-\frac{2 \pi^{2} n^{2}}{t}\right) e^{-3 t}\right] P_{3}+\left(\Pi_{-}+\Pi_{+} e^{-4 t}\right)\left(1-\frac{2 \pi^{2} n^{2}}{t}\right)\right\}
\end{aligned}
$$


Finally, by taking the trace we obtain from eq. (5.25) the heat trace

$$
\begin{aligned}
\Theta_{L_{2}}^{S^{3}}(t)= & \frac{\sqrt{\pi}}{4} t^{-3 / 2} e^{2 \lambda t} \sum_{n=-\infty}^{\infty} \exp \left(-\frac{\pi^{2} n^{2}}{t}\right) \\
& \times\left\{\left(1-\frac{2 \pi^{2} n^{2}}{t}\right) e^{3 t}+2\left(1-2 t-\frac{2 \pi^{2} n^{2}}{t}\right) e^{2 t}\right. \\
& +\left(1-\frac{2 \pi^{2} n^{2}}{t}\right) e^{t}+\left(3-16 t-\frac{6 \pi^{2} n^{2}}{t}\right) e^{-t} \\
& \left.\left.+2\left(1-2 t-\frac{2 \pi^{2} n^{2}}{t}\right) e^{-2 t}+\left(1-\frac{2 \pi^{2} n^{2}}{t}\right) e^{-3 t}\right)\right\} .
\end{aligned}
$$

We can rewrite this in terms of the function $\Omega$ as

$$
\begin{aligned}
\Theta_{L_{2}}^{S^{3}}(t)=\frac{\sqrt{\pi}}{4} t^{-3 / 2} e^{2 \lambda t}\{ & {\left[e^{3 t}+2(1-2 t) e^{2 t}+e^{t}+(3-16 t) e^{-t}+2(1-2 t) e^{-2 t}+e^{-3 t}\right] \Omega(t) } \\
& \left.-2 t\left[e^{3 t}+2 e^{2 t}+e^{t}+3 e^{-t}+2 e^{-2 t}+e^{-3 t}\right] \Omega^{\prime}(t)\right\}
\end{aligned}
$$

To second order in $t$, the exponentials may be expanded

$$
\Theta_{L_{2}}^{S^{3}}(t)=\frac{\sqrt{\pi}}{4} t^{-3 / 2}\left[10+(-26+20 \lambda) t+\left(35-52 \lambda+20 \lambda^{2}\right) t^{2}+O\left(t^{3}\right)\right] .
$$

By using the identities (5.190)-(5.191) and

$$
(3-16 t) \Omega(t)-6 t \Omega^{\prime}(t)=\frac{2 t^{3 / 2}}{\sqrt{\pi}} \sum_{n \in \mathbb{Z}}\left(3 n^{2}-8\right) e^{-t n^{2}},
$$

the heat trace can be rewritten in the spectral form

$$
\begin{gathered}
\Theta_{L_{2}}^{S^{3}}(t)=\frac{1}{2} e^{2 \lambda t} \sum_{n \in \mathbb{Z}} e^{-t n^{2}}\left\{n^{2} e^{3 t}+2\left(n^{2}-1\right) e^{2 t}+n^{2} e^{t}+\left(3 n^{2}-8\right) e^{-t}\right. \\
\left.+2\left(n^{2}-1\right) e^{-2 t}+n^{2} e^{-3 t}\right\}
\end{gathered}
$$

The asymptotic behavior of the heat trace in the limit $t \rightarrow \infty$ is

$$
\Theta_{L_{2}}^{S^{3}}(t)=e^{2 \lambda t}\left[1+O\left(e^{-3 t}\right)\right]
$$

\section{Effective action}

Now, by using (5.17), (5.18), the heat trace of quantum gravity (2.28) on $S^{1} \times S^{3}$ takes the form

$$
\Theta_{\mathrm{GR}}(t)=a_{1} \sqrt{\frac{\pi}{t}} \Omega\left(\frac{t}{a_{1}^{2}}\right)\left\{\Theta_{L_{2}}^{S^{3}}\left(\frac{t}{a^{2}}\right)-2 \Theta_{L_{1}}^{S^{3}}\left(\frac{t}{a^{2}}\right)\right\}
$$


It will be convenient to separate the asymptotic behavior at $t \rightarrow 0$

$$
\Theta_{\mathrm{GR}}(t)=\frac{\pi}{4} \frac{a_{1} a^{3}}{t^{2}} \Omega\left(\frac{t}{a_{1}^{2}}\right) W\left(\frac{t}{a^{2}}\right),
$$

where

$$
W(t)=\frac{4}{\sqrt{\pi}} t^{3 / 2}\left\{\Theta_{L_{2}}^{S^{3}}(t)-2 \Theta_{L_{1}}^{S^{3}}(t)\right\} .
$$

Note that the function $W$ depends also on the radius $a$ through the dimensionless cosmological constant $\lambda=a^{2} \Lambda$. The asymptotics of the function $W$ as $t \rightarrow 0$ are

$$
W(t)=c_{0}+c_{1} t+c_{2} t^{2}+O\left(t^{3}\right)
$$

where the coefficients $c_{k}$ are computed from eqs. (5.189) and (5.200)

$$
\begin{aligned}
& c_{0}=2, \\
& c_{1}=-46+20 \lambda, \\
& c_{2}=9-52 \lambda+20 \lambda^{2} .
\end{aligned}
$$

The coefficients $c_{k}$ differ from the coefficients $C_{k},(2.39)$, by the volume factor $\operatorname{vol}\left(S^{1} \times\right.$ $\left.S^{3}\right)=4 \pi^{3} a_{1} a^{3}$ and a uniform factor $a^{2 k}$. The asymptotics of the function $W$ as $t \rightarrow \infty$ are

$$
W(t)=\frac{4}{\sqrt{\pi}} t^{3 / 2}\left\{e^{2 \lambda t}-8 e^{t}-14+O\left(e^{-4 t}\right)+O\left(e^{(2 \lambda-3) t}\right)\right\}
$$

Now, following [12] the one-loop effective action can be presented in the form

$$
\Gamma_{(1)}=-\frac{\pi}{8} \frac{a_{1}}{a}\left\{\beta \log \frac{\mu^{2}}{\mu_{0}^{2}}+\Phi\right\},
$$

where

$$
\begin{aligned}
\Phi & =a^{4} \int_{0}^{\infty} \frac{d t}{t^{3}} e^{-t z^{2}}\left\{\Omega\left(\frac{t}{a_{1}^{2}}\right) W\left(\frac{t}{a^{2}}\right)-R_{\mathrm{GR}}\left(\frac{t}{a^{2}}\right)\right\}, \\
R_{\mathrm{GR}}(t) & =e^{-t \mu_{0}^{2}}\left\{2+\left(c_{1}+2 \mu_{0}^{2}\right) t+\left(c_{2}+c_{1} \mu_{0}^{2}+\mu_{0}^{4}\right) t^{2}\right\}, \\
\beta & =c_{2}-z^{2} a^{2} c_{1}+z^{4} a^{4},
\end{aligned}
$$

$z$ is an infrared regularization parameter, and $\mu_{0}$ is an arbitrary renormalization parameter.

The total effective action including the classical term in the one-loop approximation is

$$
\Gamma=\frac{\pi^{2}}{2 G} a_{1} a(-3+\lambda)-\hbar \frac{\pi}{8} \frac{a_{1}}{a}\left\{\beta \log \frac{\mu^{2}}{\mu_{0}^{2}}+\Phi\right\}+O\left(\hbar^{2}\right) .
$$

We neglect the terms of order $\hbar^{2}$ and set $\hbar=1$. 


\section{Thermodynamics}

The effective action is a function of two variables, $\Gamma=\Gamma\left(a_{1}, a\right)$, where $a_{1}$ is the radius of the circle $S^{1}$ and $a$ is the radius of the 3 -sphere $S^{3}$. The temperature $T$ is determined by the radius of the circle $a_{1}$ by $T=1 /\left(2 \pi a_{1}\right)$ and the spatial volume $V$ of the system is the volume of the sphere $S^{3}$, equal to $V=2 \pi^{2} a^{3}$. We introduce a dimensionless temperature

$$
x=\frac{a}{a_{1}},
$$

so that the the temperature is

$$
T=\frac{x}{2 \pi a} .
$$

Then for a canonical statistical ensemble with fixed $T$ and $V$ the free energy $F$ is determined by the effective action $\Gamma$ by

$$
F=T \Gamma=\frac{x}{2 \pi a} \Gamma .
$$

By using the results of the previous section for the effective action we obtain the free energy

$$
F=\frac{\pi}{4 G} a(-3+\lambda)-\frac{1}{16 a}\left(\beta \log \frac{\mu^{2}}{\mu_{0}^{2}}+\Phi(x, a)\right) .
$$

This enables one to compute all other thermodynamic parameters of the graviton gas such as the entropy

$$
S=-\frac{\partial F}{\partial T}=-2 \pi a \partial_{x} F,
$$

the energy

$$
E=F+T S=F-x \partial_{x} F,
$$

the pressure

$$
P=-\frac{\partial F}{\partial V}=-\frac{1}{6 \pi^{2} a^{2}} \frac{\partial F}{\partial a},
$$

and the heat capacity at constant volume

$$
C_{v}=\frac{\partial E}{\partial T}=-T \frac{\partial^{2} F}{\partial T^{2}}=-2 \pi a x \partial_{x}^{2} F .
$$

We see that the classical term and the renormalization term in the free energy (7.4) do not depend on the temperature; therefore, the entropy and the heat capacity do not depend on those terms. Therefore, the entropy and the heat capacity at constant volume are given by the derivatives of the function $\Phi$,

$$
\begin{aligned}
S & =\frac{\pi}{8} \partial_{x} \Phi, \\
C_{v} & =\frac{\pi}{8} x \partial_{x}^{2} \Phi .
\end{aligned}
$$

By changing the integration variable $t \mapsto a^{2} t$ we rewrite the function $\Phi$ as

$$
\Phi=\int_{0}^{\infty} \frac{d t}{t^{3}} e^{-t z^{2} a^{2}}\left\{\Omega\left(x^{2} t\right) W(t)-R_{\mathrm{GR}}(t)\right\} .
$$


Differentiating the function $\Phi$ with respect to $x$, we get

$$
\begin{aligned}
& \partial_{x} \Phi=2 x \int_{0}^{\infty} \frac{d t}{t^{2}} e^{-t a^{2} z^{2}} \Omega^{\prime}\left(x^{2} t\right) W(t) \\
& \partial_{x}^{2} \Phi=2 \int_{0}^{\infty} \frac{d t}{t^{2}} e^{-t a^{2} z^{2}}\left\{\Omega^{\prime}\left(x^{2} t\right)+2 x^{2} t \Omega^{\prime \prime}\left(x^{2} t\right)\right\} W(t) .
\end{aligned}
$$

We will need the asymptotics of the function $\Omega(t)$ obtained in [11]. We have as $t \rightarrow 0$

$$
\Omega(t)=1+2 \exp \left(-\frac{\pi^{2}}{t}\right)+O\left(e^{-4 \pi^{2} / t}\right)
$$

and as $t \rightarrow \infty$,

$$
\Omega(t)=\frac{1}{\sqrt{\pi}}\left[t^{1 / 2}+2 t^{1 / 2} e^{-t}+O\left(e^{-4 t}\right)\right] .
$$

Because of the asymptotic behavior of $\Omega^{\prime}$ as $t \rightarrow 0$, the integrals converge at $t \rightarrow 0$. The function $W(t)$ increases exponentially at infinity with exponent $e^{t}$ or $e^{2 \lambda t}$, the function $\Phi$ has a singularity for $z^{2}<\max \left\{\frac{1}{a^{2}}, 2 \Lambda\right\}$. We may then view the maximum of these parameters as analogous to $\Lambda_{Q C D}$, an infrared cutoff below which our analysis ceases to describe this system.

When $z$ is taken to zero, the integrals (7.11) and (7.13) do not converge, and so we do not examine the free energy or entropy past this point. However, as we will see later, because the asymptotic behavior of $\Omega^{\prime}(t)+2 t \Omega^{\prime \prime}(t)$ as $t \rightarrow \infty$ is proportional to $e^{-t}$, the heat capacity may converge even in the limit $z \rightarrow 0$. We decompose $W$ according to

$$
W(t)=\frac{4}{\sqrt{\pi}} t^{3 / 2}\left(e^{2 \lambda t}-8 e^{t}-14\right)+V(t),
$$

where the function $V$ is exponentially small as $t \rightarrow \infty$. We may then split the integral for $\partial_{x}^{2} \Phi$ into four parts:

$$
\partial_{x}^{2} \Phi=I_{1}+I_{2}+I_{3}+I_{4}
$$

where

$$
\begin{aligned}
& I_{1}=\frac{8}{\sqrt{\pi}} \int_{0}^{\infty} \frac{d t}{t^{1 / 2}} e^{-t\left(a^{2} z^{2}-2 \lambda\right)}\left\{\Omega^{\prime}\left(x^{2} t\right)+2 x^{2} t \Omega^{\prime \prime}\left(x^{2} t\right)\right\} \\
& I_{2}=-\frac{64}{\sqrt{\pi}} \int_{0}^{\infty} \frac{d t}{t^{1 / 2}} e^{-t\left(a^{2} z^{2}-1\right)}\left\{\Omega^{\prime}\left(x^{2} t\right)+2 x^{2} t \Omega^{\prime \prime}\left(x^{2} t\right)\right\} \\
& I_{3}=-\frac{112}{\sqrt{\pi}} \int_{0}^{\infty} \frac{d t}{t^{1 / 2}} e^{-t a^{2} z^{2}}\left\{\Omega^{\prime}\left(x^{2} t\right)+2 x^{2} t \Omega^{\prime \prime}\left(x^{2} t\right)\right\} \\
& I_{4}=2 \int_{0}^{\infty} \frac{d t}{t^{2}} e^{-t a^{2} z^{2}}\left\{\Omega^{\prime}\left(x^{2} t\right)+2 x^{2} t \Omega^{\prime \prime}\left(x^{2} t\right)\right\} V(t) .
\end{aligned}
$$




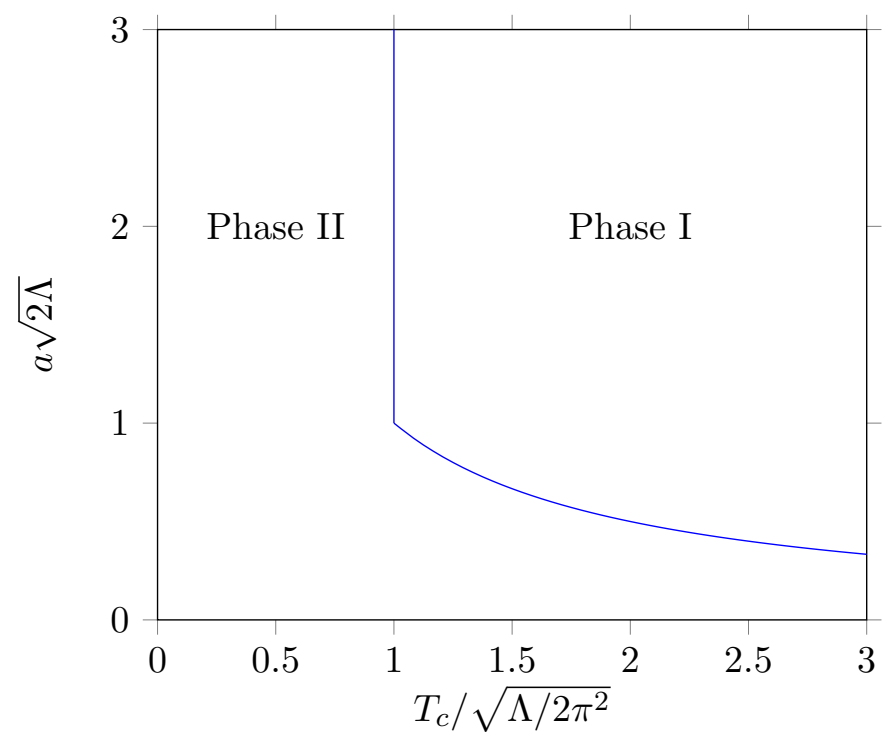

Figure 1. Phase diagram of the graviton gas.

Notice that the function in the integrand is exponentially small at infinity, namely,

$$
\Omega^{\prime}(t)+2 t \Omega^{\prime \prime}(t)=\frac{2 t^{1 / 2}}{\sqrt{\pi}}(2 t-3) e^{-t}+O\left(e^{-4 t}\right)
$$

Therefore, the integrals $I_{3}$ and $I_{4}$ converge for any $x$. The integral $I_{1}$ converges only for $x^{2}>2 \lambda-a^{2} z^{2}$ and the integral $I_{2}$ converges for $x^{2}>1-a^{2} z^{2}$. Allowing the infrared cutoff $z$ to go to zero, the integral $I_{1}$ converges only for $x^{2}>2 \lambda$ and the integral $I_{2}$ converges for $x^{2}>1$.

Therefore, all of the integrals converge at high temperature but the heat capacity has a singularity either at the temperature $x_{c}=\sqrt{2 \lambda}$ (for positive $\lambda>1 / 2$ ) or at $x_{c}=1$ if $\lambda<1 / 2$ (including the case of negative cosmological constant $\Lambda$ ). Recalling that $\lambda=a^{2} \Lambda$ and $x=2 \pi a T$, this defines the critical temperature

$$
T_{c}=\sqrt{\max \left\{\frac{\Lambda}{2 \pi^{2}}, \frac{1}{4 \pi^{2} a^{2}}\right\}},
$$

below which the system will undergo a phase transition. Notice that the smallest value of the critical temperature is

$$
T_{c, \text { min }}=\sqrt{\max \left\{\frac{\Lambda}{2 \pi^{2}}, 0\right\}} .
$$

The phase diagram of the graviton gas for the positive cosmological constant has the form illustrated on the graph figure 1.

We also study the high temperature limit as $x \rightarrow \infty$. The asymptotic behavior of the combination of derivatives of $\Omega$ (7.22) implies that the high temperature limit corresponds to the limit of $t \rightarrow \infty$. We find the limit of $I_{1}$ through $I_{3}$ replacing the $\Omega$ functions by their 
leading asymptotics and integrating:

$$
\begin{aligned}
& I_{1} \sim-\frac{16}{\pi x}, \\
& I_{2} \sim \frac{128}{\pi x}, \\
& I_{3} \sim \frac{224}{\pi x} .
\end{aligned}
$$

The integral $I_{4}$ is evaluated by changing variables $t \rightarrow t / x^{2}$ and using $V(0)=W(0)=2$; we get

$$
I_{4} \sim 4 x^{2} \nu
$$

where $\nu$ is the constant defined by the integral

$$
\nu=\int_{0}^{\infty} \frac{d t}{t^{2}}\left\{\Omega^{\prime}(t)+2 t \Omega^{\prime \prime}(t)\right\} .
$$

The integral $I_{4}$ dominates in the high temperature limit and determines the heat capacity per volume

$$
\frac{C_{v}}{V} \sim \frac{\nu_{1}}{4 \pi a^{3}} x^{3}=2 \pi^{2} \nu T^{3} .
$$

The $T^{3}$ dependence is characteristic of the photon gas and, as has been found in our previous paper [12], of the gluon gas as well.

Next we study the behavior of the heat capacity near the critical temperature. By using eq. (7.22) and setting $z=0$ we get

$$
\begin{aligned}
& I_{1} \sim \frac{32 x^{3}}{\pi} \int_{0}^{\infty} d t t e^{-t\left(x^{2}-2 \lambda\right)}, \\
& I_{2} \sim-\frac{256 x^{3}}{\pi} \int_{0}^{\infty} d t t e^{-t\left(x^{2}-1\right)} .
\end{aligned}
$$

We obtain, as $x \rightarrow \sqrt{2 \lambda}^{+}$,

$$
I_{1} \sim \frac{32 \lambda}{\pi}(x-\sqrt{2 \lambda})^{-1}
$$

and as $x \rightarrow 1^{+}$,

$$
I_{2} \sim-\frac{128}{\pi}(x-1)^{-1}
$$

The critical exponent of $(-1)$ is indicative of a second-order phase transition.

The temperature at which the phase transition occurs depends on the value of the cosmological constant. In the case that $\Lambda>\left(2 a^{2}\right)^{-1}$, the phase transition occurs at the temperature $T_{c}=\sqrt{\Lambda /\left(2 \pi^{2}\right)}$ and if $\Lambda \leq\left(2 a^{2}\right)^{-1}$ (also if $\Lambda<0$ ) the phase transition occurs at the temperature $T_{c}=1 /(2 \pi a)$. The asymptotics of the heat capacity near the critical temperature are: if $\Lambda>\left(2 a^{2}\right)^{-1}$ then as $T \rightarrow T_{c}$

$$
C_{v} \sim \frac{2^{3 / 2}}{\pi} a^{2} \Lambda^{3 / 2}\left(T-T_{c}\right)^{-1}
$$



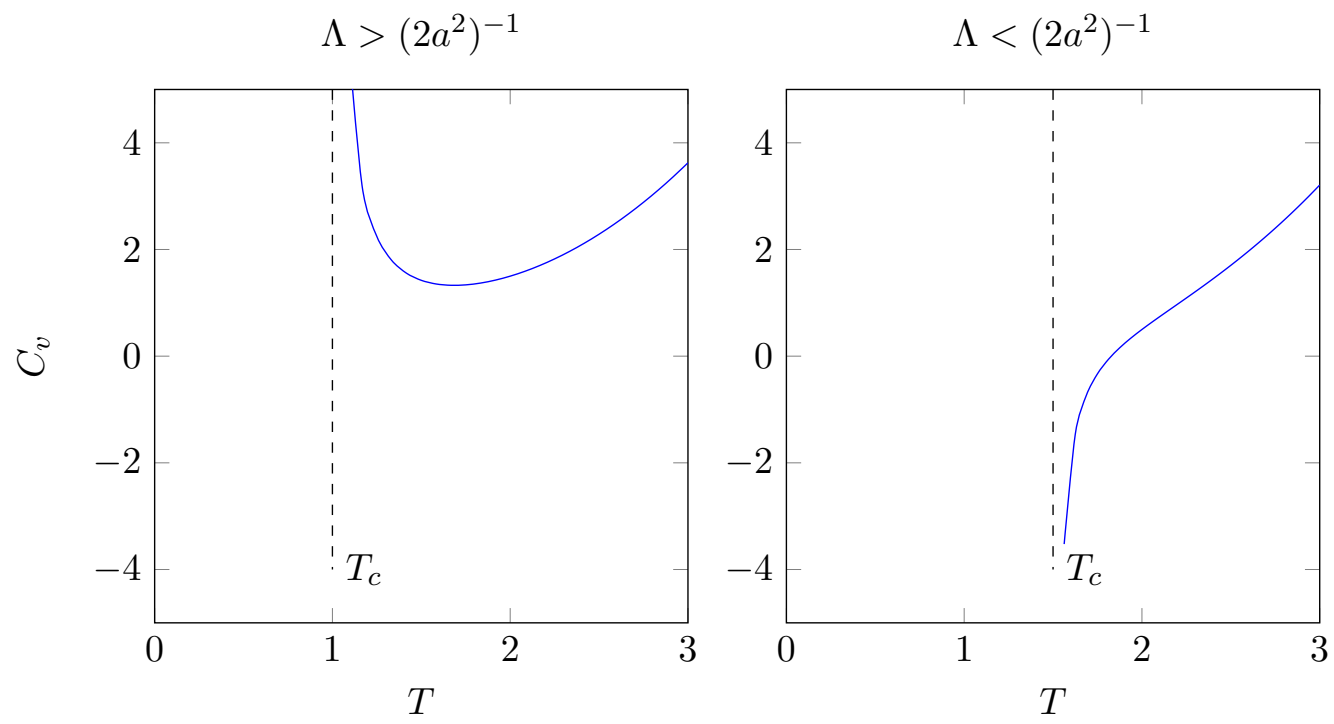

Figure 2. Heat capacity as a function of temperature.

with $T_{c}=\sqrt{\Lambda /\left(2 \pi^{2}\right)}$. Since the heat capacity at constant volume as a function of temperature grows at infinity, this means that the heat capacity must have a minimum at some temperature $T_{1}>T_{c}$. Further, if $\Lambda<\left(2 a^{2}\right)^{-1}$ then as $T \rightarrow T_{c}$

$$
C_{v} \sim-\frac{8}{\pi a}\left(T-T_{c}\right)^{-1}
$$

with $T_{c}=1 /(2 \pi a)$; in the case that $\Lambda=\left(2 a^{2}\right)^{-1}$ we have

$$
C_{v} \sim-\frac{7}{\pi a}\left(T-T_{c}\right)^{-1},
$$

with $T_{c}=1 /(2 \pi a)$. This means that the heat capacity must vanish at some temperature $T_{2}>T_{c}$ (see figure 2.).

\section{Discussion}

It is well-known that the gravitational action is unbounded from below and unstable. The primary goal of this paper was to study the quantum gravitational field restricted to a set of manifolds which have an action that is classically bounded from below, and then examine how one-loop quantum effects disturb that stability. In order to calculate the one-loop effective action exactly, it is necessary to study a spacetime with a great degree of symmetry. We studied the thermal Einstein universe $S^{1} \times S^{3}$ with non-zero cosmological constant, varying the model only with respect to the radii, $a_{1}$ and $a$, of the circle $S^{1}$ and the sphere $S^{3}$ respectively. This spacetime is off-shell, so, strictly speaking, it does depend on the gauge of the quantum field. However, we used the generally accepted minimal covariant De Witt's gauge in which all operators become Laplace type.

We computed the exact trace of the heat kernels of all relevant operators, which enabled us to calculate the one-loop effective action exactly. The lowest value of the of the graviton 
operator can be chosen to be positive by adjusting the cosmological constant, but the ghost operator always yields a negative eigenvalue, indicating an unstable mode for any radius of the Einstein universe. This may indicate a problem with the gauge condition and requires a detailed further study.

We also studied the thermal properties of the model. We found that while the free energy and entropy are ultraviolet divergent, the heat capacity is well-defined even in the infrared limit. In the high-temperature limit, the heat capacity of the graviton gas has a $T^{3}$ dependence which is typical of a photon gas, and has also been found in our previous paper [12] to be consistent with a gluon gas.

We also computed the asymptotics of the heat capacity near the critical temperature and found that the heat capacity has a branching singularity $\sim\left(T-T_{c}\right)^{-1}$ at a finite critical temperature $T_{c}$ given by (7.23).

In the case of negative or small positive cosmological constant, $\Lambda<\left(2 a^{2}\right)^{-1}$, the system exhibits a rather anomalous peculiar behavior with the negative heat capacity due to the presence of the unstable mode of the ghost operator. It is common in bound gravitational systems to have negative heat capacity. For instance, the temperature of a black hole decreases as heat is added to it. The fact that the heat capacity changes sign at some temperature $T_{2}$ indicates that the system has a minimum internal energy at that temperature.

It is interesting to play with the minimal value of the critical temperature given by (7.24). If we substitute the observed value of the cosmological constant, $\Lambda \sim 10^{-52} \mathrm{~m}^{-2}$, then the minimum critical temperature is approximately

$$
T_{c, \min }=\frac{\hbar c}{k_{B}} \sqrt{\frac{\Lambda}{2 \pi^{2}}} \sim 5 \times 10^{-4} K
$$

One can speculate that if the universe cools below the critical temperature, it is likely that some degrees of freedom would be frozen leaving a cosmic background thermal graviton radiation with temperature $T_{c}$.

The techniques used in this paper are very general. It would be interesting to extend this model to higher-derivative quantum gravity or to supergravity, in which the one-loop action vanishes on-shell.

Open Access. This article is distributed under the terms of the Creative Commons Attribution License (CC-BY 4.0), which permits any use, distribution and reproduction in any medium, provided the original author(s) and source are credited.

\section{References}

[1] I.G. Avramidi, A New algebraic approach for calculating the heat kernel in gauge theories, Phys. Lett. B 305 (1993) 27 [INSPIRE].

[2] I.G. Avramidi, The Heat kernel on symmetric spaces via integrating over the group of isometries, Phys. Lett. B 336 (1994) 171 [hep-th/9509079] [INSPIRE]. 
[3] I.G. Avramidi, Covariant algebraic calculation of the one-loop effective potential in non-Abelian gauge theory and a new approach to stability problem, J. Math. Phys. 36 (1995) 1557 [gr-qc/9403035] [INSPIRE].

[4] I.G. Avramidi, Covariant algebraic method for calculation of the low-energy heat kernel, J. Math. Phys. 36 (1995) 5055 [hep-th/9503132] [INSPIRE].

[5] I.G. Avramidi, A New algebraic approach for calculating the heat kernel in quantum gravity, J. Math. Phys. 37 (1996) 374 [hep-th/9406047] [INSPIRE].

[6] I.G. Avramidi, One-loop effective potential in higher-dimensional Yang-Mills theory, Fortsch. Phys. 47 (1999) 433 [hep-th/9604160] [INSPIRE].

[7] I.G. Avramidi, Heat Kernel and Quantum Gravity, Springer, Berlin Germany (2000).

[8] I.G. Avramidi, Heat Kernel on Homogeneous Bundles over Symmetric Spaces, Commun. Math. Phys. 288 (2009) 963 [math/0701489] [INSPIRE].

[9] I.G. Avramidi, Non-perturbative Effective Action in Gauge Theories and Quantum Gravity, Adv. Theor. Math. Phys. 14 (2010) 309 [arXiv:0903.1295] [INSPIRE].

[10] I.G. Avramidi, Mathemathical tools for calculation of the effective action in quantum gravity, in New Paths Towards Quantum Gravity, B. Booss-Bavnbek, G. Esposito and M. Lesch eds., Springer, Berlin Germany (2010), pp. 193-259.

[11] I.G. Avramidi and S. Collopy, Effective Action and Phase Transitions in Thermal Yang-Mills Theory on Spheres, Commun. Math. Phys. 311 (2012) 713 [arXiv: 1012.2414] [inSPIRE].

[12] I.G. Avramidi and S. Collopy, Thermal Yang-Mills Theory In the Einstein Universe, J. Phys. A 45 (2012) 374009 [arXiv:1201.5163] [inSPIRE].

[13] S.M. Christensen and M.J. Duff, Quantizing Gravity with a Cosmological Constant, Nucl. Phys. B 170 (1980) 480 [InSPIRE].

[14] B.S. DeWitt, The Space-time Approach to Quantum Field Theory, in Relativity, Groups and Topology II, B.S. DeWitt and R. Stora eds., Elsevier, Amsterdam Netherlands (1984), pp. $381-738$.

[15] B. DeWitt, The Global Approach to Quantum Field Theory, Oxford University Press, Oxford U.K. (2003).

[16] G.W. Gibbons and M.J. Perry, Quantizing Gravitational Instantons, Nucl. Phys. B 146 (1978) 90 [inSPIRE].

[17] P.B. Gilkey, The Spectral geometry of a Riemannian manifold, J. Diff. Geom. 10 (1975) 601 [INSPIRE].

[18] S. Hawking, The path-integral approach to quantum gravity, in General Relativity, S. Hawking and W. Israel eds., Cambridge University Press, Cambridge U.K. (1979), pp. 746-789.

[19] E. Witten, (2+1)-Dimensional Gravity as an Exactly Soluble System, Nucl. Phys. B 311 (1988) 46 [inSPIRE].

[20] E. Witten, Three-Dimensional Gravity Revisited, arXiv:0706.3359 [INSPIRE]. 